\title{
Prognostic implications of metabolism-associated gene signatures in colorectal cancer
}

\author{
Yandong Miao ${ }^{\text {Equal first author, } 1}{ }^{1}$, Qiutian Li ${ }^{\text {Equal first author, }{ }^{2} \text {, Jiangtao Wang }}{ }^{1}$, Wuxia Quan ${ }^{3}$, Chen Li $^{4}$, Yuan Yang ${ }^{1}$, Denghai \\ Mi Corresp. 1,5 \\ ${ }^{1}$ The First Clinical Medical College, Lanzhou University, Lanzhou City, Gansu Province, PR China \\ 2 Department of Oncology, 920th Hospital of Joint Logistics Support Force, Kunming City, Yunnan Province, PR China \\ 3 Qingyang people's hospital, Qingyang City, Gansu Province, PR China \\ 4 The 3rd Affiliated Hospital, Kunming Medical College,Tumor Hospital of Yunnan Province, Kunming City, Yunnan Province, PR China \\ 5 Gansu Academy of Traditional Chinese Medicine, Lanzhou City, Gansu Province, PR China \\ Corresponding Author: Denghai Mi \\ Email address: mi.dh@outlook.com
}

Colorectal cancer (CRC) is one of the most common and deadly malignancies. Novel biomarkers for the diagnosis and prognosis of this disease must be identified. Besides, metabolism plays an essential role in the occurrence and development of CRC. This article aims to identify some critical prognosis-related metabolic genes (PRMGs) and construct a prognosis model of CRC patients for clinical use. We obtained the expression profiles of CRC from The Cancer Genome Atlas database (TCGA), then identified differentially expressed PRMGs by R and Perl software. Hub genes were filtered out by univariate Cox analysis and least absolute shrinkage and selection operator Cox analysis. We used functional enrichment analysis methods, such as Gene Ontology, Kyoto Encyclopedia of Genes and Genomes, and Gene Set Enrichment Analysis, to identify involved signaling pathways of PRMGs. The nomogram predicted overall survival (OS) Calibration traces were used to evaluate the consistency between the actual and the predicted survival rate. Finally, a prognostic model was constructed based on six metabolic genes (NAT2, XDH, GPX3, AKR1C4, SPHK1, and ADCY5), and the risk score was an independent prognostic prognosticator. Genetic expression and risk score were significantly correlated with clinicopathologic characteristics of CRC. A nomogram based on the clinicopathological feature of CRC and risk score accurately predicted the OS of individual CRC cancer patients. We also validated the results in the independent colorectal cancer cohorts GSE39582 and GSE87211. Our study demonstrates that the risk score is an independent prognostic biomarker and is closely correlated with the malignant clinicopathological characteristics of CRC patients. We also determined some metabolic genes associated with the survival and clinical stage of CRC as potential biomarkers for CRC diagnosis and treatment. 


\section{Prognostic implications of metabolism-associated gene signatures in}

\section{2 colorectal cancer}

3

4 Yandong Miao ${ }^{1 *}$, Qiutian $\mathrm{Li}^{2 *}$, Jiangtao Wang ${ }^{1}$, Wuxia Quan ${ }^{3}$, Chen $\mathrm{Li}^{4}$, Yuan Yang ${ }^{1}$, Denghai $\mathrm{Mi}^{1,5}$

$5{ }^{1}$ The First Clinical Medical College of Lanzhou University, Lanzhou City, Gansu Province, PR China

$6 \quad{ }^{2}$ Department of Oncology, 920th Hospital of Joint Logistics Support Force, Kunming City, Yunnan

7 Province, PR China

$8{ }^{3}$ Qingyang People's Hospital, Qingyang City, Gansu Province, PR China

$9{ }^{4}$ The 3rd Affiliated Hospital of Kunming Medical University, Tumor Hospital of Yunnan Province,

10 Kunming City, Yunnan Province, PR China

$11{ }^{5}$ Gansu Academy of Traditional Chinese Medicine, Lanzhou City, Gansu Province, PR China

$12 *$ Equal contribution

13 Corresponding Author:

14 Deng Hai $\mathrm{Mi}^{1,5}$

$15{ }^{1}$ No.1 donggang west road, chengguan district, Lanzhou City, Gansu Province, PR China

$16{ }^{5}$ No. 418 guazhou road, qilihe district, Lanzhou City, Gansu Province, PR China

17 Email address: mi.dh@outlook.com

\section{Abstract}

Colorectal cancer (CRC) is one of the most common and deadly malignancies. Novel biomarkers for the diagnosis and prognosis of this disease must be identified. Besides, metabolism plays an essential role in the occurrence and development of CRC. This article aims to identify some critical prognosis-related metabolic genes (PRMGs) and construct a prognosis model of CRC patients for clinical use. We obtained the expression profiles of CRC from The Cancer Genome Atlas database (TCGA), then identified differentially expressed PRMGs by R and Perl software. Hub genes were filtered out by univariate Cox analysis and least absolute shrinkage and selection operator Cox analysis. We used functional enrichment analysis methods, such as Gene Ontology, Kyoto Encyclopedia of Genes and Genomes, and Gene Set Enrichment Analysis, to identify involved signaling pathways of PRMGs. The nomogram predicted overall survival (OS) Calibration traces were used to evaluate the consistency between the actual and the predicted survival rate. Finally, a prognostic model was constructed based on six metabolic genes (NAT2, XDH, 
32 Genetic expression and risk score were significantly correlated with clinicopathologic characteristics of

33 CRC. A nomogram based on the clinicopathological feature of CRC and risk score accurately predicted the 34 OS of individual CRC cancer patients. We also validated the results in the independent colorectal cancer cohorts GSE39582 and GSE87211. Our study demonstrates that the risk score is an independent prognostic biomarker and is closely correlated with the malignant clinicopathological characteristics of CRC patients. We also determined some metabolic genes associated with the survival and clinical stage of CRC as potential biomarkers for CRC diagnosis and treatment.

39

\section{Introduction}

Cancer is associated with multiple genes, and the accumulation of molecular modifications in the genome of somatic cells is the basis of cancer progression (Vogelstein \& Kinzler 2004). Cancers of all types are the primary cause of death globally and one of the most significant obstacles to increased life expectancy (World Health Organization, 2018). Colorectal cancer (CRC) is the fourth most fatal carcinoma, accounting for almost 900,000 deaths annually (Dekker et al. 2019). It is the second most frequently diagnosed cancer in women and the third most frequently diagnosed in men (Christopher J, 2018). The development of CRC is a complicated biological process requiring a constellation of factors, including lifestyle, obesity, and environmental influences that may be associated with $\mathrm{CRC}$, and which involves profound shifts at various molecular levels, including in the genome, transcriptome, methylation, and epigenome. The application of second-generation DNA sequencing techniques through whole-genome, whole-exome, and wholetranscriptome approaches leads to significant advances in cancer genomics (Meyerson et al. 2010). The Cancer Genome Atlas (TCGA) has created an unprecedented opportunity to investigate cancer biology with clinical significance (Liu et al. 2018).

Cellular metabolism is the foundation of all biological activities and participates in the regulation of cell growth and proliferation (Zhu \& Thompson 2019). Cancer cells reprogram their metabolism to utilize nitrogen and carbon to synthesize the macromolecules necessary for the growth and proliferation of tumors (Keshet et al. 2018). As mentioned before, CRC is a multi-step development in which several genetic events drive the initiation and progression of CRC. Specific metabolic pathways can also affect the occurrence and progression of CRC (La Vecchia \& Sebastián 2020). Remarkably, some genetic drivers of CRC are well-known regulators of cancer metabolism, such as p53 (Labuschagne et al. 2018), KRas (Kawada et al. 2017), and Wnt (Thompson 2014). A recent report also manifested that knockdown of MYC in CRC cells can reset the altered metabolism and suppressed cell growth (Satoh et al. 2017). Oncogene signals drive the progression of CRC associated with the control of specific metabolic pathways in CRC (Hutton et al. 2016; 
Pate et al. 2014; Satoh et al. 2017). Metastatic CRC cells participate in a selective metabolic adaptation to efficiently form liver metastasis (Loo et al. 2015). Metabolic genes also play essential role in the epithelialmesenchymal transition (Shaul et al. 2014). Previous research in metabolic genes related to CRC has garnered significant attention, and metabolic gene variants promote colorectal carcinogenesis (Hlavata et al. 2010; Hong et al. 2018; Pommier et al. 2016). Transcription and genomic data from many tumor samples have been used to research tumor metabolism and explore the role of genes to promote metabolic reprogramming in tumors (Arif et al. 2017; Dejure \& Eilers 2017; Ying et al. 2012). However, few studies have focused on constructing a prognostic model based on differential metabolic genes combined with the prognostic prediction model in CRC.

We attempted to construct a prognostic model of prognosis-related metabolic genes (PRMGs) and explore the prognostic value of these PRMGs in CRC. The colorectal cancer-specific prognostic model was used to determine some pivotal PRMGs for the diagnosis and treatment of CRC and to identify novel potential targets. We also validated the PRMG signature in an independent colorectal cancer cohort. We identified a new essential marker (risk score) to predict the prognosis of CRC patients. Our study developed a new risk model as an independent prognostic biomarker in risk stratification for CRC patients. The flow chart of the study design and analysis is shown in Figure 1.

\section{Materials and Methods}

\section{Data sources}

Both the RNA-sequencing datasets and clinical data of CRC were obtained from the TCGA database (Data Release 22.0 - January 16, 2020, https://tcga- data.nci.nih.gov/tcga/). The two main filter criteria for our data were as follows: (1) The keywords of cases are "colon, rectosigmoid junction, and rectum [Primary Site]," “"TCGA[Program],” “TCGA-COAD, TCGA-READ [Project],”, “Adenomas and Adenocarcinomas [Disease Type]."(2) The keywords of files are "Transcriptome Profiling [Data Category]," “'Gene Expression Quantification [Data Category]," "RNA-Seq [Experimental Strategy],"” “HTSeq - FPKM [Workflow Type]””), "Clinical [Data Category]," "BCR XML [Data Format]”"). The matrix files of RNA-sequencing for different samples were collated and annotated onto the genome. The expression of mRNA was extracted from the matrix file obtained from the RNA-sequencing data. The data access used GSE39582 and GSE87211 as the validation data obtained from Gene Expression Omnibus (GEO, https://www.ncbi.nlm.nih.gov/geo/ ). Raw data of gene chips are normalized using the 
RMA algorithm provided by "limma" (Ritchie et al. 2015). Perl and R-package "sva" were used to merge microarray data and reduce heterogeneity between the three studies. Metabolism-related genes were obtained from KEGG symbols of the Gene Set Enrichment Analysis (GSEA) website (https://www.gseamsigdb.org ). R software (version 3.6.2) was used for data annotation and the extraction of metabolic gene expression for the TCGA and GEO data.

\section{Identification of prognosis-associated metabolism-related genes}

Data extraction and integration were performed using Perl software. We screened differentially expressed metabolism-related genes using the Wilcox Test with R-package “edgeR”(Robinson et al. 2010), and "limma". $\mid$ Log Fold Change $\mid>1.5$ and False Discovery Rate $(F D R)<0.05$ were set as the cutoff. Bidirectional hierarchical clustering was analyzed and we drew a heatmap using R-package "pheatmap"(https://cran.r-project.org/web/packages/pheatmap/). R-package "ggplot2" was used to draw a volcano map. Prognosis-associated metabolism-related genes were identified by univariate Cox regression analysis.

\section{Construction prognosis model of metabolism-related genes and survival analysis}

We screened out the metabolism-related genes that had a significant correlation with overall survival (OS) of colorectal cancer cohorts using univariate Cox regression analysis $(\mathrm{p}<0.01)$. The least absolute shrinkage and selection operator (Lasso) Cox regression analysis constructed the optimal model of metabolism-related genes using the R-package "glmnet" (Friedman et al. 2010). Genes expression and survival analysis were evaluated using the Kaplan-Meier method and the log-rank test $(\mathrm{p}<0.05)$. The risk score for each patient calculated as: Risk score $=\sum_{j=1}^{n} \operatorname{Coefj} * X j$, Coef $j$ denotes the coefficient and $X j$ denotes the expression levels of each metabolism-related gene (Liu et al. 2019). Survival data were selected from each sample obtained from the clinical data downloaded from the TCGA and combined with the previously acquired expression profiling data. The median risk score was selected as a cutoff value to create colorectal cancer cohorts. The survival curve was drawn according to the high and lowrisk value by R-package "survival” "survminer". The R-package "survival ROC” drew the Receiver Operating Characteristic (ROC) curve, which was used to investigate the sensitivity and specificity of the survival prediction by the gene marker risk score (Le et al. 2019). Area Under Curve (AUC) served as an indicator of prognostic veracity (Le 2019; Sachs 2017). The risk curve, survival state diagram, and heatmap were drawn based on the different risk scores of the patients. Independent prognostic metabolic genes were recognized using univariate and multivariate Cox proportional risk regression analysis. We 
128

129

130

131

132

133

134

135

136

137

138

139

140

141

142

143

144

145

146

147

148

149

150

151

152

153

154

155

156

157

158

conducted clinical correlation an analysis using the R-package "beeswarm". The prognostic metabolismrelated gene was verified in the independent colorectal cancer cohort of GEO (GSE39582, GSE87211) (Hu et al. 2018; Marisa et al. 2013).

\section{Functional enrichment analysis of metabolism-related genes}

Functional enrichment analysis of metabolic genes was conducted based on the DAVID database (https://david.ncifcrf.gov/home.jsp) (Huang et al. 2009), which identified Gene Ontology (GO) categories in the biological processes (BP), cellular components (CC), or molecular functions (MF). The DAVID database was also used to determine the Kyoto Encyclopedia of Genes and Genomes (KEGG) pathways. FDR $<0.05$ was the cutoff value. R-package "GOplot" and "ggplot2" were used to integrate expression data and functional and pathway enrichment analysis. Gene Set Enrichment Analysis (GSEA) was also used to uncover the signaling pathways and biological processes of differentially expressed genes between the high and low-risk subgroups. The number of permutations was set to 1,000 , and FDR $<0.25$ was considered to be statistically significant.

\section{Exploitation of the nomogram}

Age, gender, stage, TNM stage, and risk score were used to draw a nomogram using the R-packages "Hmisc," "lattice," "Formula," "ggplot2," "foreign" and "rms". Calibration traces were used to assess the consistency between the actual and predicted survival rates. The Consistency index ( $\mathrm{C}$-index), ranging from 0.5 to 1.0 , was calculated to evaluate the model's capability for predicting an accurate prognosis. Measurements of 0.5 and 1.0 from the model represent a random probability or an excellent performance for predicting survival, respectively.

Statistical analysis was performed using the R software (Version 3.6.2; https://www.R-project.org). Pvalues for all of the analyses were: less than 0.05 (statistically significant), 0.01 (more statistically significant), and 0.001 (most statistically significant).

\section{Results}

\section{Characteristics of patients}

The TCGA CRC cohort consisted of 624 patients. Patients who have not survival time and TNM stage were excluded, resulting in a total of 596 patients. Patients were further screened, and those whose survival time was less than 90 days were also excluded, resulting in a total of 545 patients. A total of 692

Peer) reviewing PDF | (2020:03:47137:1:1:NEW 27 Jul 2020) 
mRNA expression profiles of CRC were downloaded from TCGA. Among them, 51 (7.4\%) derive from healthy samples, and $641(92.6 \%)$ come from tumor samples.

The Marisa cohort consisted of 585 patients (GSE39582), including 566 with stage I-IV colon adenocarcinoma and 19 colon mucosa samples (Marisa et al. 2013). The Hu cohort included 363 patients (GSE87211), consisting of 203 rectal tumors and 160 rectal mucosa samples (Hu et al. 2018). A total of 948 patients were obtained after merging the two sets of data. Patients with incomplete information were excluded, resulting in a total of 720 tumor patients. Patients were further screened and those whose survival time was less than 90 days were also excluded, resulting in a total of 701 tumor patients. The demographic and clinical characteristics of patients are listed in Supplementary Table 1.

\section{Construction prognosis model for TCGA colorectal cancer cohort}

A total of 102 differentially expressed metabolism-associated genes were screened containing 40 upregulated and 62 down-regulated genes. Gene expression profiles of 102 genes were displayed separately for colorectal cancer cohorts in normal and tumor tissues in Supplementary Figure 1. Univariate Cox regression analysis revealed seven metabolism-related genes that were significantly related to the OS of CRC. NAT2 and XDH were low-risk PRMGs (Hazard Ratio $(H R)<1, p<0.05$ ); GPX3, AOC3, AKR1C4, SPHK1, and ADCY5 were high-risk PRMGs (HR>1, p<0.05) (Figure 2A).

Six PRMGs were filtered out by LASSO COX regression analysis and the coefficient was calculated. The model provided the best figure when six genes were included (Figure $2 \mathrm{~B}, \mathrm{C}$ ). The coefficient of each CRC gene is shown in Figure 2D. The prognosis model was constructed based on these six genes (NAT2, XDH, GPX3, AKR1C4, SPHK1, ADCY5). The full names, pathways, and coefficients of these genes are shown in Table 1.

We investigated genetic alterations in the role of CRC risk-related metabolic genes in CRC using Gene Set Cancer Analysis (GSCA) Lite (http://bioinfo.life.hust.edu.cn/web/GSCALite/). The genes of interest in CRC were changed in 38 of 38 queried samples (100\%) (Figure 2E). The frequent genetic changes indicated the crucial roles of these genes in the development of CRC.

Each patient's risk score was calculated based on the mRNA expression level and risk coefficient of each gene. The calculation of the risk score was: risk score $=0.0283 \times$ expression of AKR1C4 $+0.0075 \times$ expression of SPHK1 $+0.0110 \times$ expression of GPX3 $+(-0.0631) \times$ expression of NAT2 $+(-0.0293) \times$ expression of $\mathrm{XDH}+0.1465 \times$ expression of ADCY5. The risk score was used to predict prognosis. 
192

193

194

195

196

197

198

199

200

201

202

203

204

205

206

207

208

209

210

211

212

213

214

215

216

217

218

219

220

221

222

223

224

Patients were divided into a high-risk group and a low-risk group with the median risk score as the cut-off value. A heatmap was developed to show the gene expression profiles of the high- and low-risk TCGACRC groups (Figure 3A) and the GEO-CRC group (Figure 3B). The risk score had a significant correlation with age, T, N, M, and clinical stage in TCGA-CRC, and age, gender, T, N, and clinical stage in GEO-CRC. The high-risk genes (GPX3, AKR1C4, SPHK1, ADCY5) were more likely to be expressed in the high-risk group patients, while low-risk genes (NAT2 and XDH) were expressed in the low-risk group patients of TCGA-CRC (Figure 3A) and GEO-CRC (Figure 3B). The association between survival time and risk score is shown in Figure 3C (TCGA-CRC), 3D (GEO-CRC). The survival time decreased as risk score increased and the higher the risk score, the more deaths in TCGA-CRC (Figure 3E) and GEOCRC (Figure 3F). Risk scores both were significantly related to OS in the univariate independent prognostic analysis of TCGA-CRC $(\mathrm{HR}=3.324,95 \% \mathrm{CI}=1.956-5.650, \mathrm{P}<0.001)$ (Figure $3 \mathrm{G})$ and GEO$\mathrm{CRC}(\mathrm{HR}=2.096,95 \% \mathrm{CI}=1.383-3.177, \mathrm{P}<0.001)$ (Figure $3 \mathrm{H})$.

The multivariate independent prognostic analysis showed that the risk score was an independent prognostic predictor in the TCGA-CRC group ( $\mathrm{HR}=2.639,95 \% \mathrm{CI}=1.413-4.928, \mathrm{P}=0.002)$ (Figure 4A) and the GEO-CRC group ( $\mathrm{HR}=1.658,95 \% \mathrm{CI}=1.059-2.598, \mathrm{P}=0.027)$ (Figure 4B). Kaplan-Meier cumulative curves indicated that the OS rate between the high-risk group and the low-risk group was significantly different. The survival time of patients with high-risk score was significantly shorter than that of patients with low-risk score in the TCGA-CRC group $(\mathrm{P}<0.001)$ (Figure 4C) and GEO-CRC group $(\mathrm{P}<0.001)$ (Figure 4D). The 1-, 3-, and 5-year AUC values of the risk score were 0.672, 0.608, and 0.648, respectively, and the prognostic accuracy of the stage was higher than other clinical characteristics in TCGA-CRC (Figure 4E, 4G, 4I). Similarly, the 1-, 3-, and 5-year AUC values of the risk score were $0.612,0.566$, and 0.573 , respectively, and the prognostic accuracy of the M-stage was higher than other clinical characteristics in GEO-CRC (Figure 4F, 4H, 4J). These results confirm that six metabolic gene signatures can also predict survival in the independently validated GEO colorectal cohort.

\section{The risk score and metabolic genes related to the clinicopathological features of CRC}

The expressions of NAT2, ADCY5, SPHK1, GPX3, and the risk score were significantly correlated with the clinicopathological features of TCGA-CRC (Supplementary Table 2, Figure 5A-5K). NAT2 was highly expressed in stages I, II, $\mathrm{N}_{0}$, and $\mathrm{M}_{0}$, but had low expression in stages III, IV, $\mathrm{N}_{1-2}$, and $\mathrm{M}_{1}$ (Figure $5 A-5 C)(p<0.05)$. However, the expression level of ADCY5 was higher in stages III, IV, and $\mathrm{N}_{1-2}$ than in stages I, II, and $\mathrm{N}_{0}$ (Figure 5D, 5E). SPHK1 and GPX3 were also highly expressed in $\mathrm{T}_{3-4}$ but had low expressions in $\mathrm{T}_{1-2}$ (Figure $5 \mathrm{~F}, 5 \mathrm{G}$ ). The risk score was significantly correlated with the clinical stage, 
225

226

227

228

229

230

231

232

233

234

235

236

237

238

239

240

241

242

243

244

245

246

247

248

249

250

251

252

253

254

255

256

257

which was highly expressed in stages III, IV, $\mathrm{T}_{3-4}, \mathrm{~N}_{1-2}$, and $\mathrm{M}_{1}$, but had low expression in stages I, II, $\mathrm{T}_{1-}$. ${ }_{2}, \mathrm{~N}_{0}$, and $\mathrm{M}_{0}$, (Figure $\left.5 \mathrm{H}-5 \mathrm{~K}\right)(\mathrm{p}<0.05)$. These results showed that NAT2 was a low-risk metabolic gene, and ADCY5, SPHK1, GPX3, were high-risk metabolic genes, which is consistent with our previous results. More importantly, the risk score closely correlated with the malignant clinicopathological characteristics of CRC and is an independent prognostic factor.

Similar results were seen in the validation group (Supplementary Table 3, Figure 5L-5AA). NAT2 and $\mathrm{XDH}$ were highly expressed in the $<65$-year age group, $\mathrm{M}_{0}$, and had low expression in the $>65$-year age group, $\mathrm{M}_{1}$ (Figure 5L-5O) ( $\left.\mathrm{p}<0.05\right)$. The expression level of ADCY5 was the opposite of NAT2 and XDH (Figure 5P, 5Q). SPHK1 and GPX3 were highly expressed in stage III, IV, $\mathrm{T}_{3-4}$, and $\mathrm{N}_{1-3}$, and had low expressions in stages I, II, $\mathrm{T}_{1-2}$, and $\mathrm{N}_{0}$ (Figure $5 \mathrm{R}-5 \mathrm{~W}$ ). The expression of AKR1C4 was higher in stages III, IV, and $\mathrm{N}_{1-3}$, than in stages I, II, and $\mathrm{N}_{0}$ (Figure $5 \mathrm{X}-5 \mathrm{Y}$ ). The risk score was higher in the group of $>65$ years old and $M_{1}$, lower in the group of $<65$ years old and $M_{0}($ Figure $5 Z, 5 A A)(p<0.05)$.

\section{Identification involved signaling pathways of PRMGs}

GO enrichment analysis of the seven metabolic genes showed that there were two GO terms in BP, one GO term in MF, and one GO term in $\mathrm{CC}$, which was significant (FDR $<0.05$ ). GO enrichment analysis showed that these seven genes could be classified into several essential processes, including oxidationreduction, the xenobiotic metabolic process, electron carrier activity, and cytosol (Figure 6A). KEGG pathway enrichment shown that PRMGs were mainly enriched in caffeine metabolism, metabolic pathways, and drug metabolism by other enzymes (Figure 6B) $(p<0.05)$. GSEA analysis showed that changed genes were observably enriched in several common pathways. 98 of 178 gene sets were upregulated in the high-risk phenotype group, and 95 gene sets were remarkable at FDR $<25 \%$. The topfive gene sets of the high-risk group were significantly related to basal cell carcinoma (NES=2.31, $\mathrm{P}=0.000$ ), dilated cardiomyopathy $(\mathrm{NES}=2.28, \mathrm{P}=0.000$ ), vascular smooth muscle contraction (NES=2.25, $\mathrm{P}=0.000$ ), glycosaminoglycan biosynthesis chondroitin sulfate $(\mathrm{NES}=2.24, \mathrm{P}=0.000$ ), and axon guidance $(\mathrm{NES}=2.23, \mathrm{P}=0.000) .80$ of 178 gene sets were upregulated in the low-risk phenotype group. 74 gene sets were markedly enriched at FDR $<25 \%$, and the five most common gene sets of the low-risk group were negatively associated with peroxisome (NES=-2.37, $\mathrm{P}=0.000)$, fatty acid metabolism $(\mathrm{NES}=-2.36, \mathrm{P}=0.000)$, butanoate metabolism $(\mathrm{NES}=-2.31, \mathrm{P}=0.000)$, valine, leucine, and isoleucine degradation ( $\mathrm{NES}=-2.26, \mathrm{P}=0.000$ ), and propanoate metabolism ( $\mathrm{NES}=-2.26, \mathrm{P}=0.000)$ (Figure 6C). The integration of the 5 most prevalent phenotypes of the high-risk and the low-risk groups was visualized in Figure 6D. 
258 A characterized prognostic prediction model

259 A nomogram is a powerful tool quantifying an individual's risk in a clinical setting by integrating multiple 260 risk factors (Liang et al. 2015; Won et al. 2015). We used the nomogram to predict the probabilities of 1-, 2613 - and 5-year OS by incorporating the age, gender, TNM stage, and risk score of TCGA-CRC (Figure 262 7A). The results were verified in the GEO-CRC. Each factor was assigned a score in proportion to its 263 contribution to the risk of survival. The calibration curve showed that the actual survival time is in 264 agreement with the predicted survival time and the C-index is 0.8. (Figure 7B, 7D, 7F). A 75-year old (60 265 points) female patients (6 points) would acquire a total of 224 points if she had stage III (41 points), T3 266 (32 points), N1 (0 points), and M0 (0 points), with a risk score (85 points). Her 1-, 3-, and 5-year survival 267 rate was approximately $67 \%, 45 \%$, and $28 \%$, respectively. The nomogram in the GEO colorectal cancer 268 cohorts and the 1-, 3- and 5- year calibration curves are shown in Figure 7C, 7E, and 7G, respectively.

\section{Discussion}

271 Specific metabolic activities can directly influence the transformational process or support the biological processes that make tumors grow (Vander Heiden \& DeBerardinis 2017). Recent data have shown that microbial metabolites, such as secondary bile acids, promote carcinogenesis; metabolic links between gut microbes are associated with cancer and a diet rich in fat and meat; and extracellular metabolic energetics can promote cancer progression, especially in colorectal cancer (Jia et al. 2018; Louis et al. 2014; Wirbel et al. 2019; Wong \& Yu 2019). Prognostic predictions are crucial to the selection of clinical treatment regimens for cancer patients. Several studies have explored prognostic biomarkers and found that gene expression profiles play a vital role in the prognosis of cancer (Jiang et al. 2019; Shen et al. 2019a; Zhou et al. 2018).

We attempted to construct a prognostic model for CRC patients based on six PRMGs filtered out by LASSO COX regression analysis and determined the risk score. The prognostic model was accurate and gave a precise predictive value. Our results showed that the risk score is an independent prognostic factor. The expression of NAT2, ADCY5, SPHK1, GPX3, and risk score also was significantly associated with the clinicopathological features of CRC. The prognostic metabolism-associated gene signature was validated in an independent GEO colorectal cancer cohort. We also explored the mechanism of the seven PRMGs through GO term, KEGG pathway enrichment, and GSEA analysis. The results showed that these genes correlated with some metabolic processes and metabolic pathways. Moreover, we constructed a nomogram to predict 1-, 3- and 5-year OS validated within the independent cohort of GEO databases. 
291 Univariate Cox regression analysis demonstrated that seven metabolism-related genes were significantly related to the OS of CRC, including five high-risk PRMGs and two low-risk PRMGs. Previous reports have shown that Glutathione peroxidase 3 (GPX3) methylation may play a crucial role in predicting the platinum sensitivity of CRC(Pelosof et al. 2017). SPHK1 has an essential role in the development of multifunctional NF- $\mathrm{BB}-$ regulated cytokine IL-6 and is continuously activated by the transcription factor STAT3. It also plays a role in colitisassociated cancer (Liang et al. 2013). There are additional reports that AKR1C4 and ADCY5 are hub genes that may be independent prognosis biomarkers and therapeutic targets for CRC patients (Gylfe et al. 2013; Yang et al. 2019), which was consistent with our results.

Whether the expression level of metabolism-related genes can be used as a prognostic maker is a vital topic of research. Our colorectal cancer prognostic model based on six metabolism-related genes was found to be of value and the 1-, 3-, and 5-year risk scores and the AUC values of ROC were consistent with previous reports (Jeun et al. 2019; Park et al. 2009; Peng et al. 2019; Sun et al. 2019). Prognoses were classified as high- and low-risk and the six metabolic genes we screened may be ideal prognostic markers. 5-year OS was about 50\% in the high-risk group and 75\% in the low-risk group, which was consistent with previous reports (Dueland et al. 2018). High-risk genes in the model, including GPX3, AKR1C4, SPHK1 and ADCY5, have been reported to promote hypermethylation, rare mutations, cancer progression, poor prognosis, and the developmental process of malignant cells in CRC patient samples or cell lines (Gylfe et al. 2013; Kawamori et al. 2009; Liang et al. 2013; Pan et al. 2019; Zhou et al. 2019). SPHK1 promotes the phosphorylation and activation of $\mathrm{p} 65$, thus promoting the occurrence of CRC (Shen et al. 2019b). The expressions of NAT2, ADCY5, SPHK1, GPX3, and risk score were significantly correlated with the clinicopathological features of $\mathrm{CRC}$, and the risk score was closely correlated with the malignant clinicopathological characteristics of CRC and is an independent prognostic factor. NAT2 is associated with a high risk of colorectal cancer, mainly due to its involvement in the metabolism of aromatic and heterocyclic aromatic amines in cooked red meat (Lilla et al. 2006). We also identified six metabolically-related genes that were significantly correlated with gene expression and prognosis in CRC patients in the GEO database (a separate cohort of 720 CRC patients). GPX3, AKR1C4, and SPHK1 are reported to have involvement in the pathogenesis of $\mathrm{CRC}$ and in predicting overall survival, reinforcing the prognostic value of our TCGA and GEO cohort analysis. The remaining ADCY5 gene has not been associated with CRC prognosis and may be used as a potential biomarker for CRC. 
323

324

325

326

327

328

329

330

331

332

333

334

335

336

337

338

339

340

341

342

343

344

processes and signaling pathways have been identified by GO enrichment analysis, caffeine metabolism, metabolic pathways, and KEGG pathway analysis including oxidation-reduction and the xenobiotic metabolic process. Previous research demonstrated that the oxidation-reduction process and xenobiotic metabolic process play a crucial role in the development of CRC or colorectal cancer cells (Bensard et al. 2020; Han et al. 2016).

We constructed a nomogram to predict individual clinical outcomes. The nomogram is a stable and reliable tool for quantifying individual risk by combining and describing risk factors. It has been used for tumor prognosis, including for CRC (Renfro et al. 2017; Sjoquist et al. 2018). The nomogram generates a graphical statistical prediction model that assigns scores to each factor, including age, sex, and clinical stage. The model summarizes all clinical points to provide numerical possibilities for clinical outcomes such as OS, relapse, and drug nonadherence. In addition to traditional clinicopathologic features such as TNM staging, tumor size, and histological subtypes, risk scores based on genetic markers can also be incorporated into a predictive nomogram model to predict clinical outcomes (Reichling et al. 2019; Sjoquist et al. 2018). A nomogram predicted 3- and 5 -year recurrence-free survival rates for non-small cell lung cancer and gave a prognostic score calculated by the autophagy gene signature (Liu et al. 2019). The combination of autophagy gene characteristics and prognostic factors has a better prognostic value than the single application (Mo et al. 2019). Calibration curves showed that the nomogram, which included RNA signals and conventional prognostic factors, accurately predicted 3 - and 5-year survival probabilities (Xiong et al. 2017). Our nomogram, which includes risk scores and clinicopathologic features, is a good predictor of survival in 1 -, 3 - and 5-year CRC patients.

We constructed a six metabolic gene model for colorectal cancer patients based on TCGA to predict the prognosis of colorectal cancer patients. A risk score based on six genes may be a promising independent prognostic biomarker and is closely correlated with the malignant clinicopathological characteristics for CRC patients. A nomogram based on genetic characteristics and clinicopathologic characteristics may accurately predict the survival probability of individual CRC patients at 1,3 , and 5 years. Some of the programmatic improvements to the model can be made at other levels to represent scheduling activities in more detail. Further research on these genes may improve their clinical application and may provide a new perspective into the pathogenesis of CRC. Some of the antecedently overlooked genes may be additional biomarkers for CRC and require further study. Our study improved our understanding of the interactions between CRC cells and the tumor metabolism microenvironment and may identify novel therapeutic targets.

Peer) reviewing PDF | (2020:03:47137:1:1:NEW 27 Jul 2020) 
355 Conclusion

356 We comprehensively identified PRMGs, constructed a six-metabolic gene model, and analyzed their molecular

357

358

359

360

361

362

363

364

365

366

367

368

369

370

371

372

373

374

375

376

377

378

379

380

381

382

383

384

385

386

387

388

389

function in CRC. Our study also highlighted the crucial role of the risk score as an independent prognostic

biomarker that is closely correlated with the malignant clinicopathological characteristics for CRC patients. Our research identified several crucial processes and signaling pathways of the metabolic genes in CRC. These findings provide a comprehensive outlook for further studies into the roles of metabolic genes in the pathogenesis of CRC and as potential biomarkers for CRC diagnosis and therapeutics.

\section{Acknowledgements}

We thank the TCGA and GEO databases for the availability of the data.

\section{References}

Arif T, Krelin Y, Nakdimon I, Benharroch D, Paul A, Dadon-Klein D, and Shoshan-Barmatz V. 2017. VDAC1 is a molecular target in glioblastoma, with its depletion leading to reprogrammed metabolism and reversed oncogenic properties. Neuro Oncol 19:951964. 10.1093/neuonc/now297

Bensard CL, Wisidagama DR, Olson KA, Berg JA, Krah NM, Schell JC, Nowinski SM, Fogarty S, Bott AJ, Wei P, Dove KK, Tanner JM, Panic V, Cluntun A, Lettlova S, Earl CS, Namnath DF, Vazquez-Arreguin K, Villanueva CJ, Tantin D, Murtaugh LC, Evason KJ, Ducker GS, Thummel CS, and Rutter J. 2020. Regulation of Tumor Initiation by the Mitochondrial Pyruvate Carrier. Cell Metab 31:284-300.e287. 10.1016/j.cmet.2019.11.002

Christopher J . Global, regional, and national disability-adjusted life-years (DALYs) for 359 diseases and injuries and healthy life expectancy (HALE) for 195 countries and territories, 1990-2017: a systematic analysis for the Global Burden of Disease Study 2017. Lancet 392:1859-1922. 10.1016/s0140-6736(18)32335-3

Dejure FR, and Eilers M. 2017. MYC and tumor metabolism: chicken and egg. Embo j 36:34093420. 10.15252/embj.201796438

Dekker E, Tanis PJ, Vleugels JLA, Kasi PM, and Wallace MB. 2019. Colorectal cancer. Lancet 394:1467-1480. 10.1016/s0140-6736(19)32319-0

Dueland S, Foss A, Solheim JM, Hagness M, and Line PD. 2018. Survival following liver transplantation for liver-only colorectal metastases compared with hepatocellular carcinoma. Br J Surg 105:736-742. 10.1002/bjs.10769 
390

391

392

393

394

395

396

397

398

399

400

401

402

403

404

405

406

407

408

409

410

411

412

413

414

415

416

417

418

419

420

421

422

423

424

425

426

427

428

429

430

Friedman J, Hastie T, and Tibshirani R. 2010. Regularization Paths for Generalized Linear Models via Coordinate Descent. J Stat Softw 33:1-22.

Gylfe AE, Katainen R, Kondelin J, Tanskanen T, Cajuso T, Hanninen U, Taipale J, Taipale M, Renkonen-Sinisalo L, Jarvinen H, Mecklin JP, Kilpivaara O, Pitkanen E, Vahteristo P, Tuupanen S, Karhu A, and Aaltonen LA. 2013. Eleven candidate susceptibility genes for common familial colorectal cancer. PLoS Genet 9:e1003876. 10.1371/journal.pgen.1003876

Han A, Bennett N, MacDonald A, Johnstone M, Whelan J, and Donohoe DR. 2016. Cellular Metabolism and Dose Reveal Carnitine-Dependent and -Independent Mechanisms of Butyrate Oxidation in Colorectal Cancer Cells. J Cell Physiol 231:1804-1813. 10.1002/jcp.25287

Hlavata I, Vrana D, Smerhovsky Z, Pardini B, Naccarati A, Vodicka P, Novotny J, MohelnikovaDuchonova B, and Soucek P. 2010. Association between exposure-relevant polymorphisms in CYP1B1, EPHX1, NQO1, GSTM1, GSTP1 and GSTT1 and risk of colorectal cancer in a Czech population. Oncol Rep 24:1347-1353. 10.3892/or_00000992

Hong Y, Liew SC, Thean LF, Tang CL, and Cheah PY. 2018. Human colorectal cancer initiation is bidirectional, and cell growth, metabolic genes and transporter genes are early drivers of tumorigenesis. Cancer Lett 431:213-218. 10.1016/j.canlet.2018.06.005

Hu Y, Gaedcke J, Emons G, Beissbarth T, Grade M, Jo P, Yeager M, Chanock SJ, Wolff H, Camps J, Ghadimi BM, and Ried T. 2018. Colorectal cancer susceptibility loci as predictive markers of rectal cancer prognosis after surgery. Genes Chromosomes Cancer 57:140-149. 10.1002/gcc.22512

Huang DW, Sherman BT, and Lempicki RA. 2009. Systematic and integrative analysis of large gene lists using DAVID bioinformatics resources. Nature Protocols 4:44-57. 10.1038/nprot.2008.211

Hutton JE, Wang X, Zimmerman LJ, Slebos RJ, Trenary IA, Young JD, Li M, and Liebler DC. 2016. Oncogenic KRAS and BRAF Drive Metabolic Reprogramming in Colorectal Cancer. Mol Cell Proteomics 15:2924-2938. 10.1074/mcp.M116.058925

Jeun M, Lee HJ, Park S, Do EJ, Choi J, Sung YN, Hong SM, Kim SY, Kim DH, Kang JY, Son HN, Joo J, Song EM, Hwang SW, Park SH, Yang DH, Ye BD, Byeon JS, Choe J, Yang SK, Moinova H, Markowitz SD, Lee KH, and Myung SJ. 2019. A Novel Blood-Based Colorectal Cancer Diagnostic Technology Using Electrical Detection of Colon Cancer Secreted Protein-2. Adv Sci (Weinh) 6:1802115. 10.1002/advs.201802115

Jia W, Xie G, and Jia W. 2018. Bile acid-microbiota crosstalk in gastrointestinal inflammation and carcinogenesis. Nat Rev Gastroenterol Hepatol 15:111-128. 10.1038/nrgastro.2017.119

Jiang C, Wu S, Jiang L, Gao Z, Li X, Duan Y, Li N, and Sun T. 2019. Network-based approach to identify biomarkers predicting response and prognosis for HER2-negative breast cancer treatment with taxane-anthracycline neoadjuvant chemotherapy. PeerJ 7:e7515. 10.7717/peerj.7515 
431

432

433

434

435

436

437

438

439

440

441

442

443

444

445

446

447

448

449

450

451

452

453

454

455

456

457

458

459

460

461

462

463

464

465

466

467

468

469

470

Kawada K, Toda K, and Sakai Y. 2017. Targeting metabolic reprogramming in KRAS-driven cancers. Int J Clin Oncol 22:651-659. 10.1007/s10147-017-1156-4

Kawamori T, Kaneshiro T, Okumura M, Maalouf S, Uflacker A, Bielawski J, Hannun YA, and Obeid LM. 2009. Role for sphingosine kinase 1 in colon carcinogenesis. Faseb j 23:405414. 10.1096/fj.08-117572

Keshet R, Szlosarek P, Carracedo A, and Erez A. 2018. Rewiring urea cycle metabolism in cancer to support anabolism. Nat Rev Cancer 18:634-645. 10.1038/s41568-018-0054-z

La Vecchia S, and Sebastián C. 2020. Metabolic pathways regulating colorectal cancer initiation and progression. Semin Cell Dev Biol 98:63-70. 10.1016/j.semcdb.2019.05.018

Labuschagne CF, Zani F, and Vousden KH. 2018. Control of metabolism by p53 - Cancer and beyond. Biochim Biophys Acta Rev Cancer 1870:32-42. 10.1016/j.bbcan.2018.06.001

Le NQK. 2019. Fertility-GRU: Identifying Fertility-Related Proteins by Incorporating Deep-Gated Recurrent Units and Original Position-Specific Scoring Matrix Profiles. J Proteome Res 18:3503-3511. 10.1021/acs.jproteome.9b00411

Le NQK, Yapp EKY, and Yeh HY. 2019. ET-GRU: using multi-layer gated recurrent units to identify electron transport proteins. Bmc Bioinformatics 20:377. 10.1186/s12859-0192972-5

Liang J, Nagahashi M, Kim EY, Harikumar KB, Yamada A, Huang WC, Hait NC, Allegood JC, Price MM, Avni D, Takabe K, Kordula T, Milstien S, and Spiegel S. 2013. Sphingosine-1phosphate links persistent STAT3 activation, chronic intestinal inflammation, and development of colitis-associated cancer. Cancer Cell 23:107-120. 10.1016/j.ccr.2012.11.013

Liang W, Zhang L, Jiang G, Wang Q, Liu L, Liu D, Wang Z, Zhu Z, Deng Q, Xiong X, Shao W, Shi $X$, and He J. 2015. Development and validation of a nomogram for predicting survival in patients with resected non-small-cell lung cancer. J Clin Oncol 33:861-869. 10.1200/jco.2014.56.6661

Lilla C, Verla-Tebit E, Risch A, Jäger B, Hoffmeister M, Brenner H, and Chang-Claude J. 2006. Effect of NAT1 and NAT2 genetic polymorphisms on colorectal cancer risk associated with exposure to tobacco smoke and meat consumption. Cancer Epidemiol Biomarkers Prev 15:99-107. 10.1158/1055-9965.Epi-05-0618

Liu J, Lichtenberg T, Hoadley KA, Poisson LM, Lazar AJ, Cherniack AD, Kovatich AJ, Benz CC, Levine DA, Lee AV, Omberg L, Wolf DM, Shriver CD, Thorsson V, and Hu H. 2018. An Integrated TCGA Pan-Cancer Clinical Data Resource to Drive High-Quality Survival Outcome Analytics. Cell 173:400-416.e411. 10.1016/j.cell.2018.02.052

Liu Y, Wu L, Ao H, Zhao M, Leng X, Liu M, Ma J, and Zhu J. 2019. Prognostic implications of autophagy-associated gene signatures in non-small cell lung cancer. Aging (Albany NY) 11. 10.18632/aging.102544

Loo JM, Scherl A, Nguyen A, Man FY, Weinberg E, Zeng Z, Saltz L, Paty PB, and Tavazoie SF. 2015. Extracellular metabolic energetics can promote cancer progression. Cell 160:393406. 10.1016/j.cell.2014.12.018 
471 Louis P, Hold GL, and Flint HJ. 2014. The gut microbiota, bacterial metabolites and colorectal

472 cancer. Nat Rev Microbiol 12:661-672. 10.1038/nrmicro3344

Marisa L, de Reynies A, Duval A, Selves J, Gaub MP, Vescovo L, Etienne-Grimaldi MC, Schiappa R, Guenot D, Ayadi M, Kirzin S, Chazal M, Flejou JF, Benchimol D, Berger A, Lagarde A, Pencreach E, Piard F, Elias D, Parc Y, Olschwang S, Milano G, Laurent-Puig $P$, and Boige V. 2013. Gene expression classification of colon cancer into molecular subtypes: characterization, validation, and prognostic value. PLoS Med 10:e1001453. 10.1371/journal.pmed.1001453

Meyerson M, Gabriel S, and Getz G. 2010. Advances in understanding cancer genomes through second-generation sequencing. Nat Rev Genet 11:685-696. 10.1038/nrg2841

Mo S, Dai W, Xiang W, Li Y, Feng Y, Zhang L, Li Q, and Cai G. 2019. Prognostic and predictive value of an autophagy-related signature for early relapse in stages I-III colon cancer. Carcinogenesis 40:861-870. 10.1093/carcin/bgz031

Pan X, Zeng T, Yuan F, Zhang YH, Chen L, Zhu L, Wan S, Huang T, and Cai YD. 2019. Screening of Methylation Signature and Gene Functions Associated With the Subtypes of Isocitrate Dehydrogenase-Mutation Gliomas. Front Bioeng Biotechnol 7:339. 10.3389/fbioe.2019.00339

Park Y, Freedman AN, Gail MH, Pee D, Hollenbeck A, Schatzkin A, and Pfeiffer RM. 2009. Validation of a colorectal cancer risk prediction model among white patients age 50 years and older. J Clin Oncol 27:694-698. 10.1200/jco.2008.17.4813

Pate KT, Stringari C, Sprowl-Tanio S, Wang K, TeSlaa T, Hoverter NP, McQuade MM, Garner C, Digman MA, Teitell MA, Edwards RA, Gratton E, and Waterman ML. 2014. Wnt signaling directs a metabolic program of glycolysis and angiogenesis in colon cancer. Embo j 33:1454-1473. 10.15252/embj.201488598

Pelosof L, Yerram S, Armstrong T, Chu N, Danilova L, Yanagisawa B, Hidalgo M, Azad N, and Herman JG. 2017. GPX3 promoter methylation predicts platinum sensitivity in colorectal cancer. Epigenetics 12:540-550. 10.1080/15592294.2016.1265711

Peng L, Balavarca Y, Weigl K, Hoffmeister M, and Brenner H. 2019. Head-to-Head Comparison of the Performance of 17 Risk Models for Predicting Presence of Advanced Neoplasms in Colorectal Cancer Screening. Am J Gastroenterol 114:1520-1530. 10.14309/ajg.0000000000000370

Pommier AJ, Farren M, Patel B, Wappett M, Michopoulos F, Smith NR, Kendrew J, Frith J, Huby R, Eberlein C, Campbell H, Womack C, Smith PD, Robertson J, Morgan S, Critchlow SE, and Barry ST. 2016. Leptin, BMI, and a Metabolic Gene Expression Signature Associated with Clinical Outcome to VEGF Inhibition in Colorectal Cancer. Cell Metab 23:77-93. 10.1016/j.cmet.2015.10.015

Reichling C, Taieb J, Derangere V, Klopfenstein Q, Le Malicot K, Gornet JM, Becheur H, Fein F, Cojocarasu O, Kaminsky MC, Lagasse JP, Luet D, Nguyen S, Etienne PL, Gasmi M, Vanoli A, Perrier H, Puig PL, Emile JF, Lepage C, and Ghiringhelli F. 2019. Artificial intelligence-guided tissue analysis combined with immune infiltrate assessment predicts stage III colon cancer outcomes in PETACC08 study. Gut. 10.1136/gutjnl-2019-319292 
512 Renfro LA, Goldberg RM, Grothey A, Sobrero A, Adams R, Seymour MT, Heinemann V,

513

514

515

516

517

518

519

520

521

522

523

524

525

526

527

528

529

530

531

532

533

534

535

536

537

538

539

540

541

542

543

544

545

546

547

548

549

550

551

552 Schmoll HJ, Douillard JY, Hurwitz H, Fuchs CS, Diaz-Rubio E, Porschen R, Tournigand C, Chibaudel B, Hoff PM, Kabbinavar FF, Falcone A, Tebbutt NC, Punt CJA, Hecht JR, Souglakos J, Bokemeyer C, Van Cutsem E, Saltz L, de Gramont A, and Sargent DJ. 2017. Clinical Calculator for Early Mortality in Metastatic Colorectal Cancer: An Analysis of Patients From 28 Clinical Trials in the Aide et Recherche en Cancerologie Digestive Database. J Clin Oncol 35:1929-1937. 10.1200/jco.2016.71.5771

Ritchie ME, Phipson B, Wu D, Hu Y, Law CW, Shi W, and Smyth GK. 2015. limma powers differential expression analyses for RNA-sequencing and microarray studies. Nucleic Acids Research 43:e47-e47. 10.1093/nar/gkv007 \%J Nucleic Acids Research

Robinson MD, McCarthy DJ, and Smyth GK. 2010. edgeR: a Bioconductor package for differential expression analysis of digital gene expression data. Bioinformatics 26:139140. 10.1093/bioinformatics/btp616

Sachs MC. 2017. plotROC: A Tool for Plotting ROC Curves. J Stat Softw 79. 10.18637/jss.v079.c02

Satoh K, Yachida S, Sugimoto M, Oshima M, Nakagawa T, Akamoto S, Tabata S, Saitoh K, Kato K, Sato S, Igarashi K, Aizawa Y, Kajino-Sakamoto R, Kojima Y, Fujishita T, Enomoto A, Hirayama A, Ishikawa T, Taketo MM, Kushida Y, Haba R, Okano K, Tomita M, Suzuki Y, Fukuda S, Aoki M, and Soga T. 2017. Global metabolic reprogramming of colorectal cancer occurs at adenoma stage and is induced by MYC. Proc Natl Acad Sci U S A 114:E7697-e7706. 10.1073/pnas.1710366114

Shaul YD, Freinkman E, Comb WC, Cantor JR, Tam WL, Thiru P, Kim D, Kanarek N, Pacold ME, Chen WW, Bierie B, Possemato R, Reinhardt F, Weinberg RA, Yaffe MB, and Sabatini DM. 2014. Dihydropyrimidine accumulation is required for the epithelialmesenchymal transition. Cell 158:1094-1109. 10.1016/j.cell.2014.07.032

Shen S, Kong JJ, Qiu YW, Yang XW, Wang WT, and Yan LN. 2019a. Identification of core genes and outcomes in hepatocellular carcinoma by bioinformatics analysis. Journal of Cellular Biochemistry 120:10069-10081. 10.1002/jcb.28290

Shen Z, Feng X, Fang Y, Li Y, Li Z, Zhan Y, Lin M, Li G, Ding Y, and Deng H. 2019b. POTEE drives colorectal cancer development via regulating SPHK1/p65 signaling. Cell Death Dis 10:863. 10.1038/s41419-019-2046-7

Sjoquist KM, Renfro LA, Simes RJ, Tebbutt NC, Clarke S, Seymour MT, Adams R, Maughan TS, Saltz L, Goldberg RM, Schmoll HJ, Van Cutsem E, Douillard JY, Hoff PM, Hecht JR, Tournigand C, Punt CJA, Koopman M, Hurwitz H, Heinemann V, Falcone A, Porschen R, Fuchs C, Diaz-Rubio E, Aranda E, Bokemeyer C, Souglakos I, Kabbinavar FF, Chibaudel B, Meyers JP, Sargent DJ, de Gramont A, and Zalcberg JR. 2018. Personalizing Survival Predictions in Advanced Colorectal Cancer: The ARCAD Nomogram Project. J Natl Cancer Inst 110:638-648. 10.1093/jnci/djx253

Sun YH, Li J, Shu HJ, Li ZL, and Qian JM. 2019. Serum immunoinflammation-related protein complexes discriminate between inflammatory bowel disease and colorectal cancer. Clin Transl Oncol 21:1680-1686. 10.1007/s12094-019-02100-3 
Thompson CB. 2014. Wnt meets Warburg: another piece in the puzzle? Embo $j$ 33:1420-1422. 10.15252/embj.201488785

Vander Heiden MG, and DeBerardinis RJ. 2017. Understanding the Intersections between Metabolism and Cancer Biology. Cell 168:657-669. 10.1016/j.cell.2016.12.039

Vogelstein B, and Kinzler KW. 2004. Cancer genes and the pathways they control. Nat Med 10:789-799. 10.1038/nm1087

Wirbel J, Pyl PT, Kartal E, Zych K, Kashani A, Milanese A, Fleck JS, Voigt AY, Palleja A, Ponnudurai R, Sunagawa S, Coelho LP, Schrotz-King P, Vogtmann E, Habermann N, Nimeus E, Thomas AM, Manghi P, Gandini S, Serrano D, Mizutani S, Shiroma H, Shiba S, Shibata T, Yachida S, Yamada T, Waldron L, Naccarati A, Segata N, Sinha R, Ulrich CM, Brenner H, Arumugam M, Bork P, and Zeller G. 2019. Meta-analysis of fecal metagenomes reveals global microbial signatures that are specific for colorectal cancer. Nat Med 25:679-689. 10.1038/s41591-019-0406-6

Won YW, Joo J, Yun T, Lee GK, Han JY, Kim HT, Lee JS, Kim MS, Lee JM, Lee HS, Zo JI, and Kim S. 2015. A nomogram to predict brain metastasis as the first relapse in curatively resected non-small cell lung cancer patients. Lung Cancer 88:201-207. 10.1016/j.lungcan.2015.02.006

Wong SH, and Yu J. 2019. Gut microbiota in colorectal cancer: mechanisms of action and clinical applications. Nat Rev Gastroenterol Hepatol 16:690-704. 10.1038/s41575-0190209-8

World Health Organization. Global Health Observatory. Geneva: World Health Organization; 2018. who.int/gho/database/en/. Accessed June 21, 2018.

Xiong Y, Wang R, Peng L, You W, Wei J, Zhang S, Wu X, Guo J, Xu J, Lv Z, and Fu Z. 2017. An integrated IncRNA, microRNA and mRNA signature to improve prognosis prediction of colorectal cancer. Oncotarget 8:85463-85478. 10.18632/oncotarget.20013

Yang G, Zhang Y, and Yang J. 2019. A Five-microRNA Signature as Prognostic Biomarker in Colorectal Cancer by Bioinformatics Analysis. Front Oncol 9:1207. 10.3389/fonc. 2019.01207

Ying $\mathrm{H}$, Kimmelman AC, Lyssiotis CA, Hua S, Chu GC, Fletcher-Sananikone E, Locasale JW, Son J, Zhang H, Coloff JL, Yan H, Wang W, Chen S, Viale A, Zheng H, Paik JH, Lim C, Guimaraes AR, Martin ES, Chang J, Hezel AF, Perry SR, Hu J, Gan B, Xiao Y, Asara JM, Weissleder R, Wang YA, Chin L, Cantley LC, and DePinho RA. 2012. Oncogenic Kras maintains pancreatic tumors through regulation of anabolic glucose metabolism. Cell 149:656-670. 10.1016/j.cell.2012.01.058

Zhou C, Hu H, Zheng Z, Chen C, Li Y, Li B, and Duan S. 2019. Association between GPX3 promoter methylation and malignant tumors: A meta-analysis. Pathol Res Pract 215:152443. 10.1016/j.prp.2019.152443

Zhou DY, Li X, Zhao HY, Sun BH, Liu AQ, Han X, Cui ZQ, and Yuan LJ. 2018. Combining multidimensional data to identify a key signature (gene and miRNA) of cisplatin-resistant gastric cancer. Journal of Cellular Biochemistry 119:6997-7008. 10.1002/jcb.26908 
593 Zhu J, and Thompson CB. 2019. Metabolic regulation of cell growth and proliferation. Nat Rev 594 Mol Cell Biol 20:436-450. 10.1038/s41580-019-0123-5

595

596 


\section{Table 1 (on next page)}

Table1 Functions of genes in the prognostic gene signatures. 
1 Table1 Functions of genes in the prognostic gene signatures.

\begin{tabular}{|c|c|c|c|}
\hline Gene symbol & Full name & Pathway & Risk coefficient \\
\hline AKR1C4 & $\begin{array}{l}\text { Aldo-keto reductase family } \\
1 \text { member } \mathrm{C} 4\end{array}$ & $\begin{array}{l}\text { Participate in multiple hormone } \\
\text { metabolism processes }\end{array}$ & 0.0283 \\
\hline SPHK1 & Sphingosine kinase 1 & Sphingolipid metabolism & 0.0075 \\
\hline GPX3 & Glutathione peroxidase 3 & Glutathione metabolism & 0.0110 \\
\hline NAT2 & $\mathrm{N}$-acetyltransferase 2 & $\begin{array}{c}\text { Drug metabolism - cytochrome } \\
\text { P450 }\end{array}$ & -0.0631 \\
\hline $\mathrm{XDH}$ & Xanthine dehydrogenase & $\begin{array}{l}\text { Involved in the oxidative } \\
\text { metabolism of purines }\end{array}$ & -0.0293 \\
\hline ADCY5 & Adenylate cyclase 5 & $\begin{array}{l}\text { Links between intrauterine growth } \\
\text { and adult height and metabolism }\end{array}$ & 0.1465 \\
\hline
\end{tabular}

2 
Figure 1

Figure 1. A flow chart of the study design and analysis.

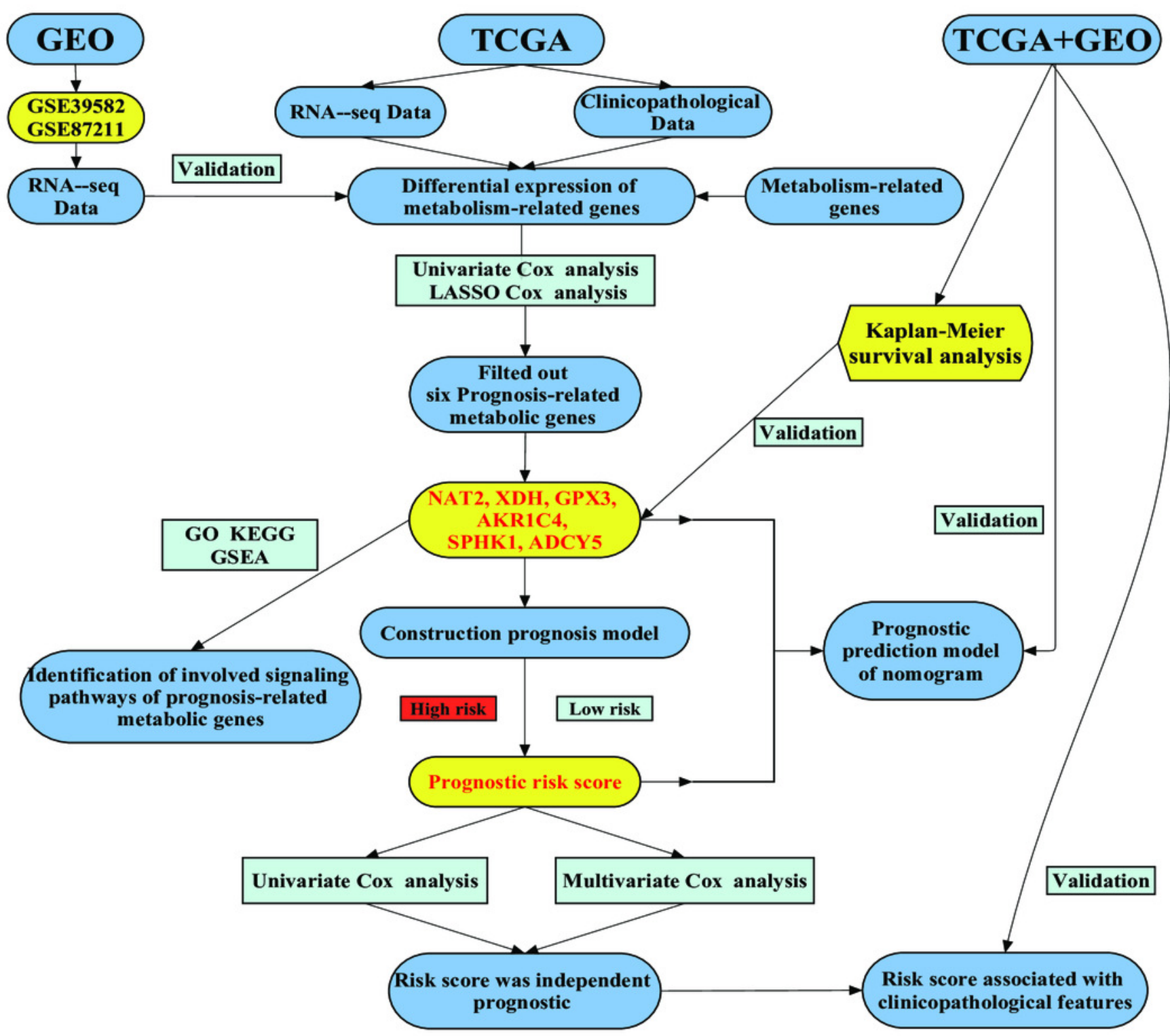




\section{Figure 2}

Figure 2. Establishment of prognostic metabolic gene signature by univariate and LASSO Cox regression analysis.

(A-D) The process of constructing the signature containing six metabolic genes. (A) The HR, 95\% Cl calculated by univariate Cox regression. A coefficient (D) profile plot was generated against the log (lambda) sequence (B). (C) Selection of the optimal parameter (lambda) in the LASSO model for colorectal cancer. (E) Genetic alteration of the six genes in the colorectal cancer cohort. $X$ axis represents cancer type, sky blue indicates COAD, light blue indicates READ. The left $Y$ axis represents ratio of gene mutation, right $Y$ axis represents gene names. Dark blue, cyan, and pink small rectangles indicate the type of gene mutation. $\mathrm{HR}$, hazard ratios; $\mathrm{Cl}$, confidence intervals; COAD, colon adenocarcinoma; READ, rectum adenocarcinoma. 
A

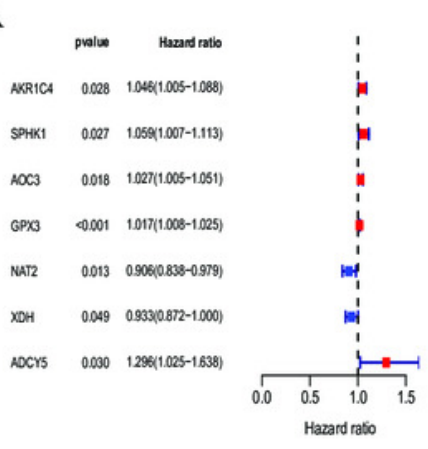

B

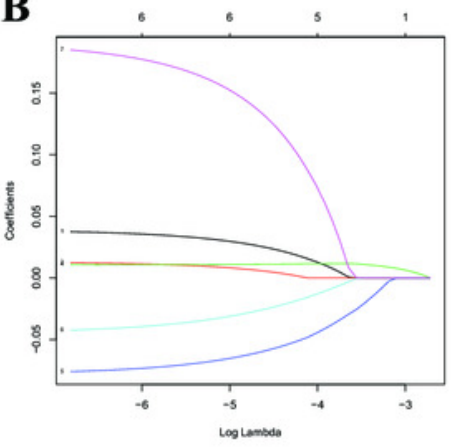

C

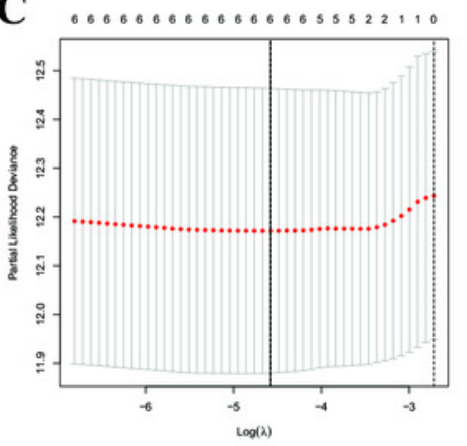

D

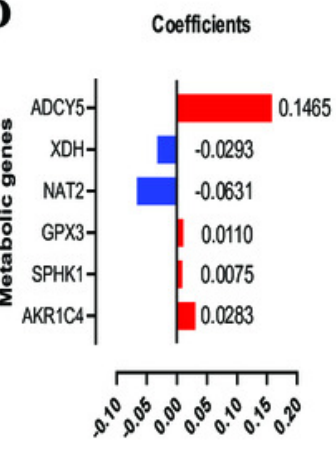

$\mathbf{E}$

Altered in $38(100 \%)$ of 38 samples.

4

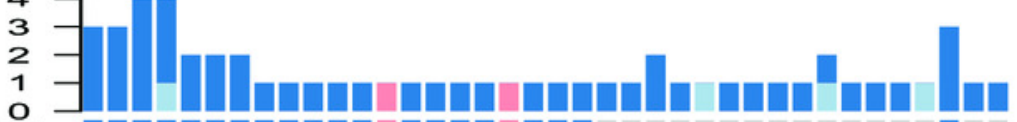

$58 \%$ \%

$32 \%$

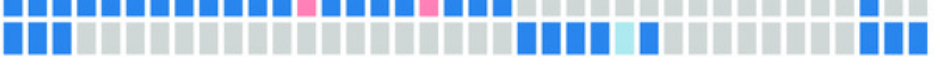

$16 \%$

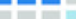

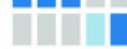

$8 \%$

$8 \%$

1.

$8 \%$
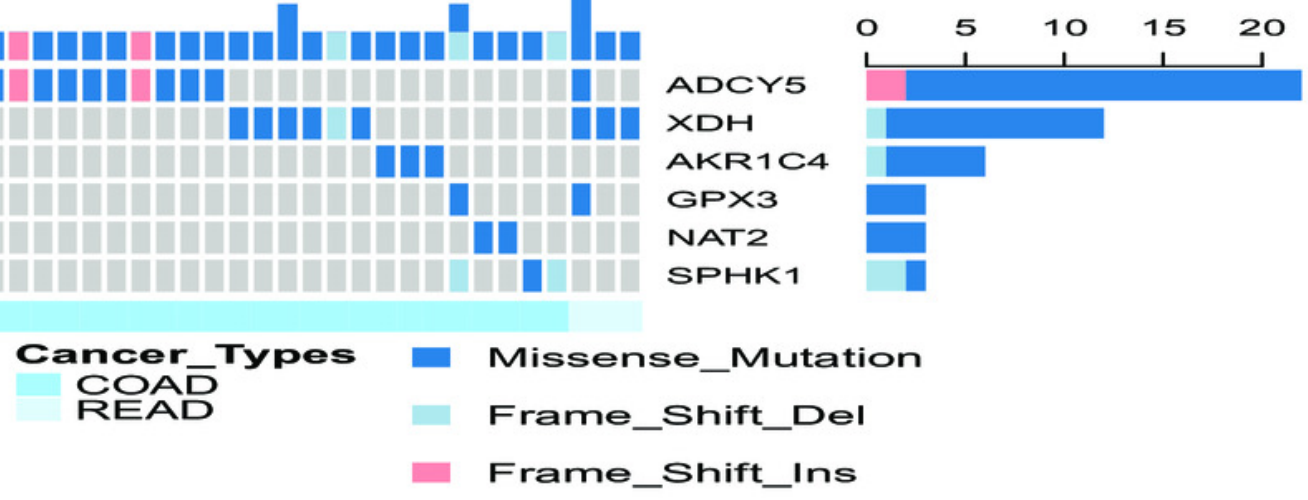

AKR1C4

GPX3

NAT2

SPHK1 


\section{Figure 3}

Figure 3. Traits of the prognostic metabolism-associated genes signature.

Heatmap of the metabolism-associated gene expression profiles in prognostic signature for TCGA-CRC (A) and GEO-CRC (B). The distribution of risk score and patient's survival time of TCGA-CRC (C) and GEO-CRC (D). The black dotted line is the optimum cutoff dividing patients into low-risk and high-risk groups. The red curve represents high risk and the blue curve represents low risk. The distribution of survival status of TCGA-CRC (E) and GEO-CRC (F). The dots indicate the survival status, the red dot indicates the death of the patient and the blue dot indicates alive. $(\mathbf{G}, \mathbf{H})$ Univariate Cox regression analysis. Forest plot of the association between risk factors and survival of TCGA-CRC (G) and GEO-CRC (H). TCGA, the cancer genome atlas database; GEO, gene expression omnibus; CRC, colorectal cancer. 


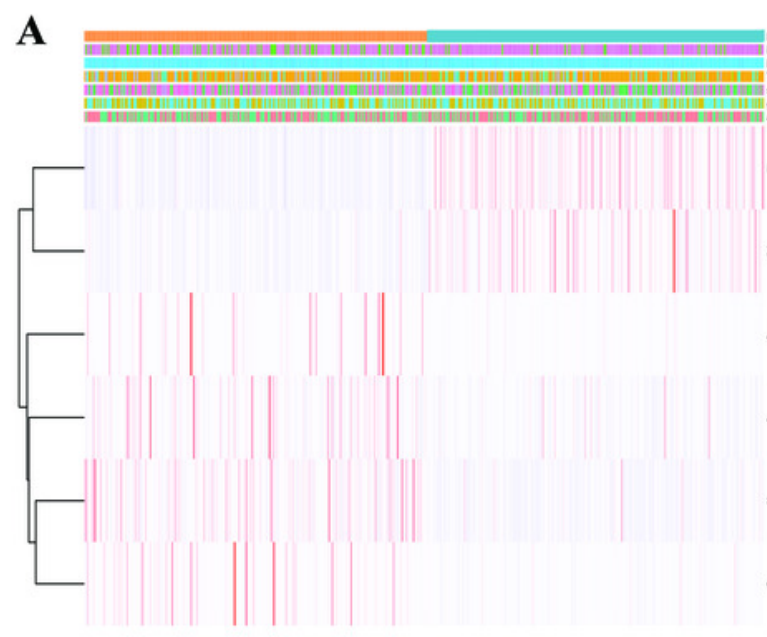

C

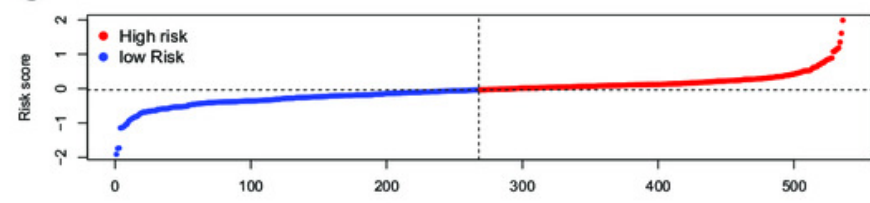

E

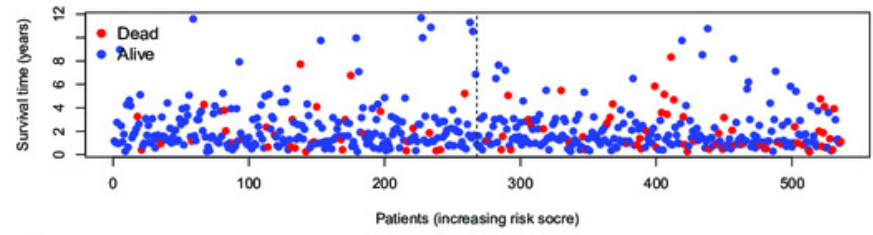

\section{G}
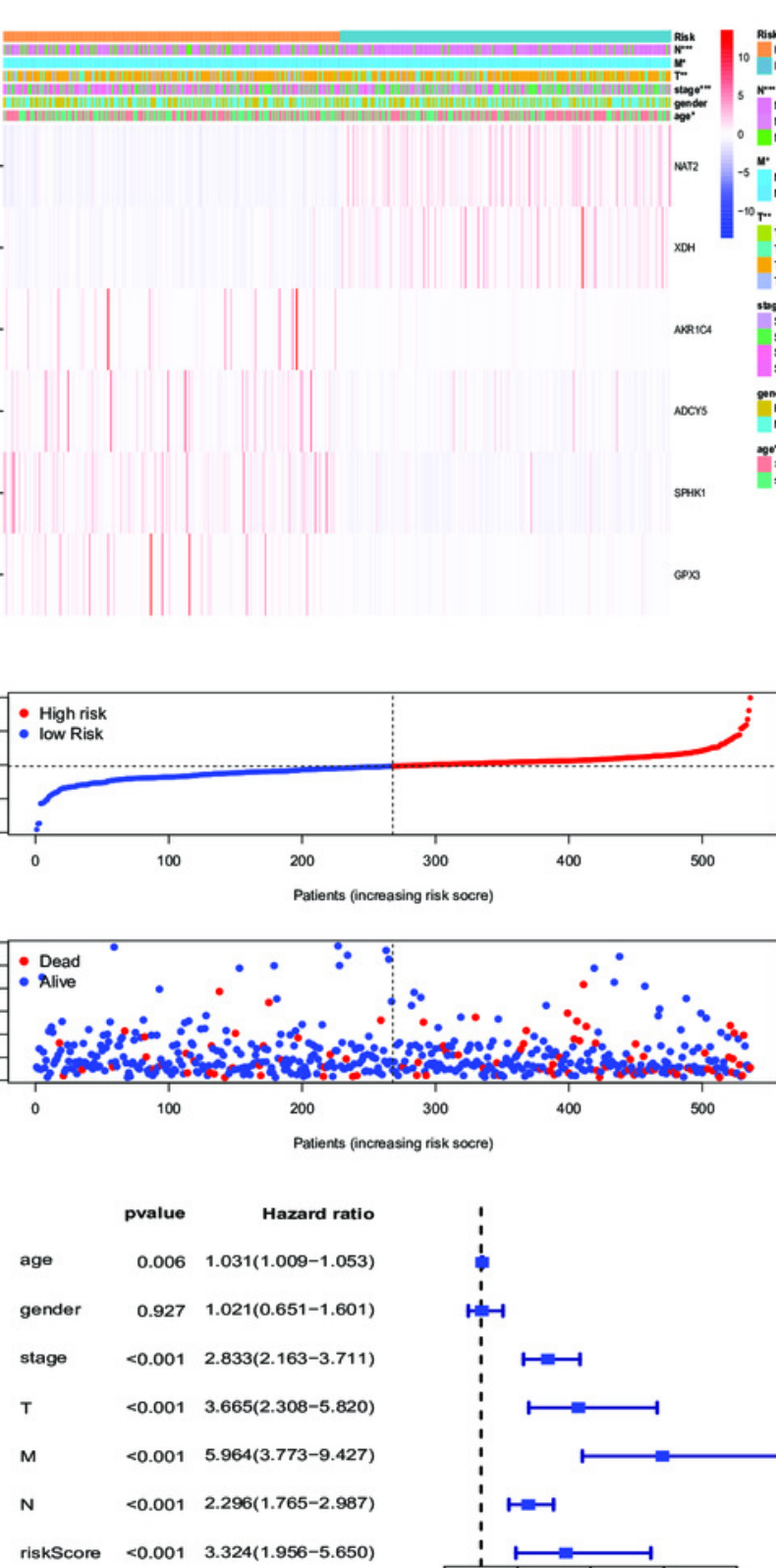

D

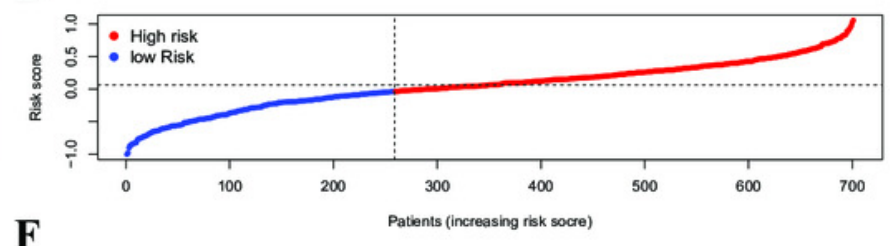

F

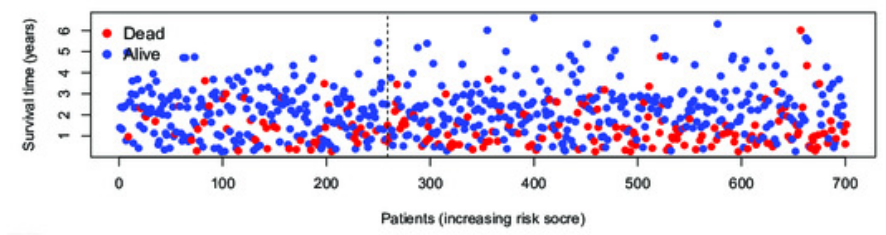

H

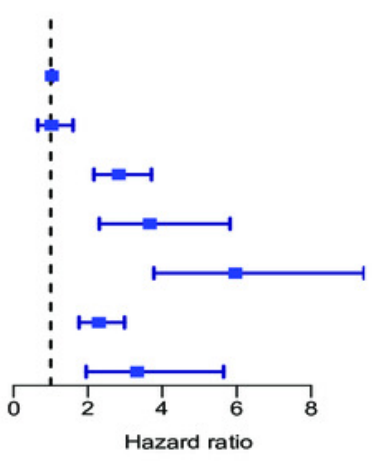




\section{Figure 4}

Figure 4. Metabolism -associated gene signature was significantly associated with survival in colorectal cancer.

(A, B) Multivariate Cox regression analysis. The risk score was an independent prognostic element in TCGA-CRC (A) and GEO-CRC (B). (C, D) Kaplan-Meier survival analysis of CRC patients ranked by the median risk score. $X$ axis represents the survival time (year) of the CRC patient, $Y$ axis represents the survival probability of the CRC patient. The high-risk score was related to poor OS in TCGA-CRC (C) and GEO-CRC (D). ROC analysis of the sensitivity and specificity of the OS for the combination of risk score and clinical characteristics in TCGACRC (E, G, I) and GEO-CRC (F, H, J). TCGA, the cancer genome atlas database; GEO, gene expression omnibus; CRC, colorectal cancer; ROC, receiver operating characteristic; OS, overall survival; AUC, area under curve; $\mathrm{T}$, primary tumor; $\mathrm{M}$, distant metastasis; $\mathrm{N}$, regional lymph nodes. 
A

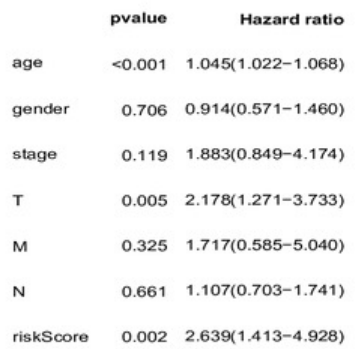

B

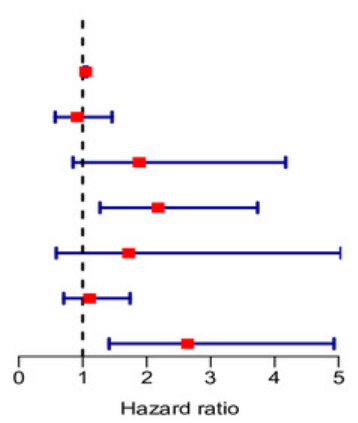

C

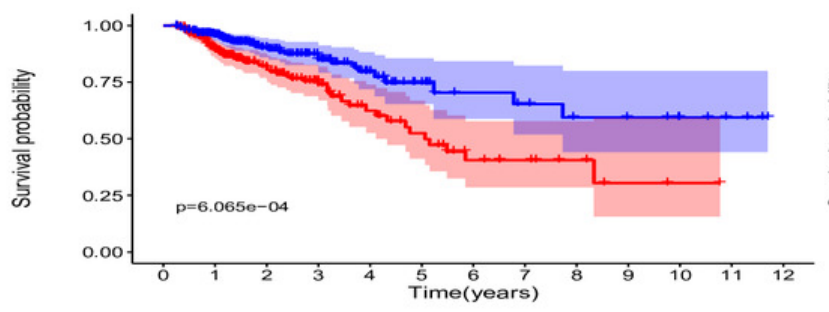

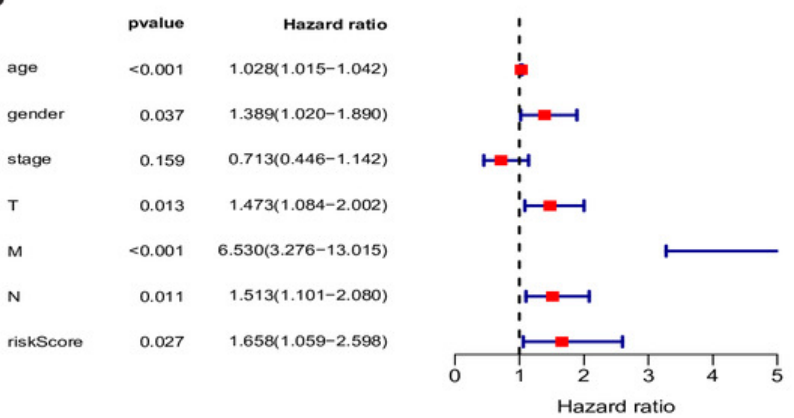

D Risk \pm High risk \pm Low risk

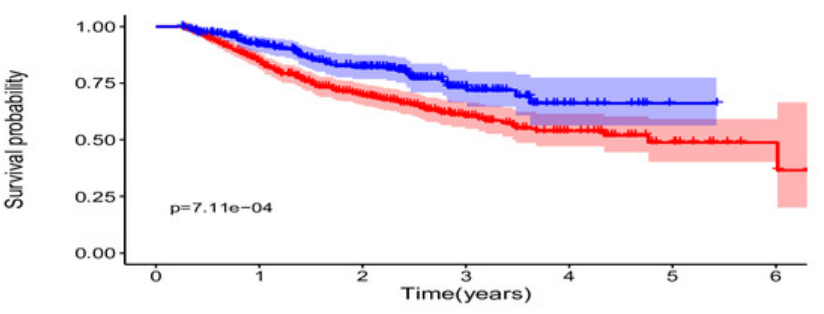

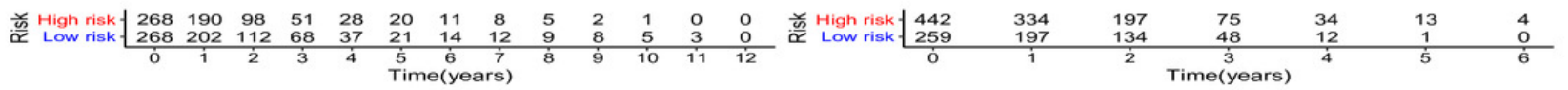

$\mathbf{E}$

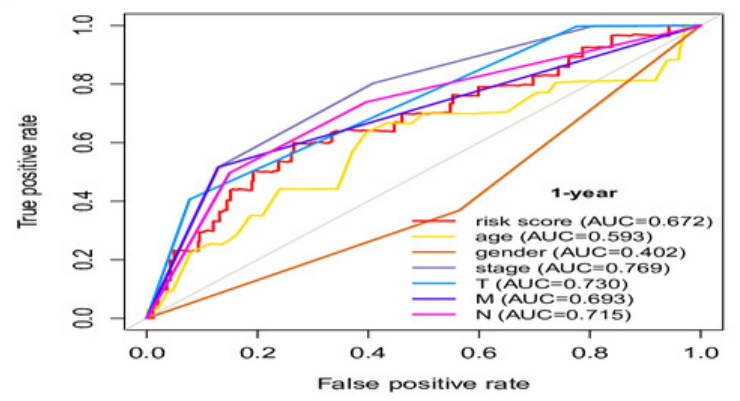

G

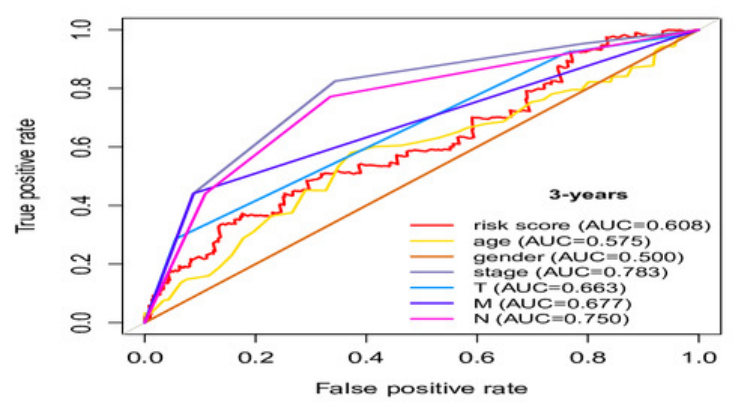

I

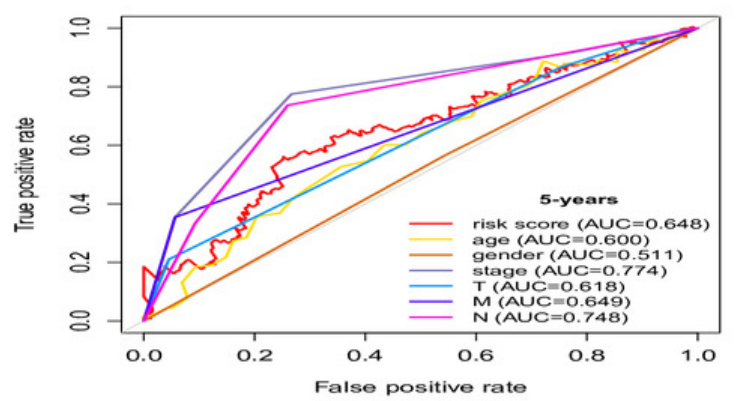

F

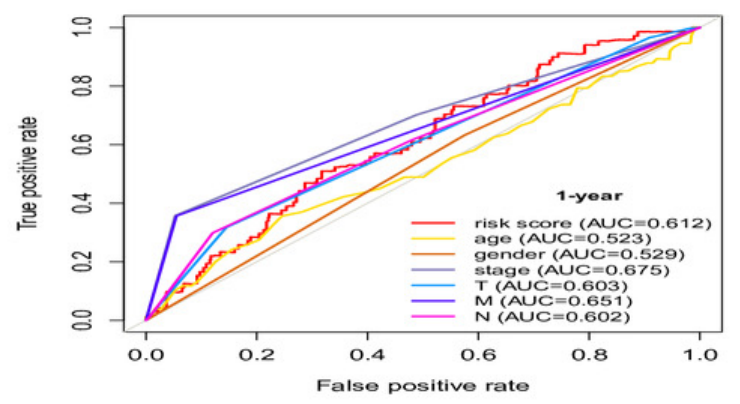

H

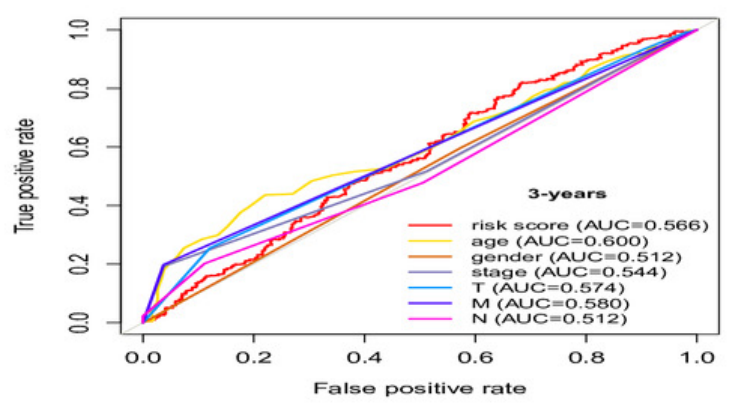

$\mathbf{J}$

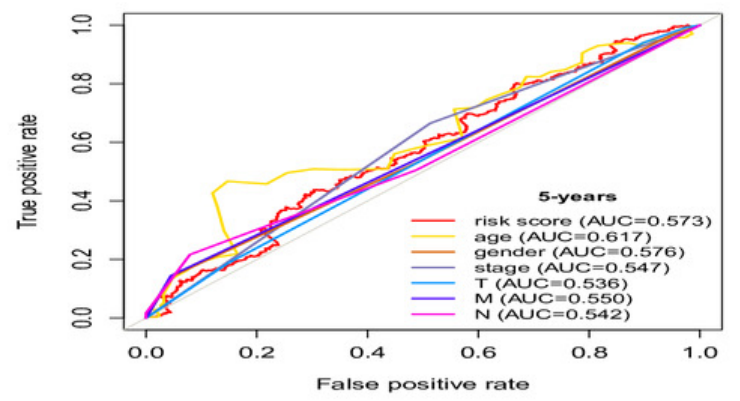




\section{Figure 5}

Figure 5. Risk score and metaboli sm-genes associated with the clinicopathological features of CRC.

Box-plot showed that there was a significant association between metabolic genes expression, risk score, and clinicopathological features in the TCGA dataset (A-K) and GEO dataset (L-AA). In the TCGA dataset, the expression of NAT2 related to the group of the stage (A), N-stage (B), and M-stage (C). The expression of ADCY5 related to stage (D) and Nstage (E); SPHK1 (F), GPX3 (G) associated with T-stage; Risk score associated with stage (H), T-stage (I), N-stage (J), and M-stage (K). In the GEO dataset, the expression of NAT2 related to a group of age (I) and M-stage (M). The expression of XDH related to a group of age (N) and M-stage (0). The expression of ADCY5 associated with age (P) and M-stage (Q); SPHK1 associated with stage (R), T-stage (S), and N-stage (T); GPX3 associated with stage (U), Tstage (V), and N-stage (W); AKR1C4 had a correlation with stage (X) and N-stage (Y). Risk score associated with age (Z) and M-stage (AA). 
TCGA

A

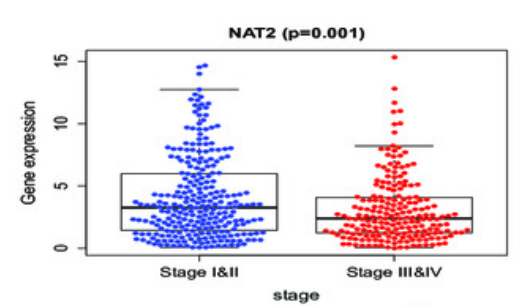

D

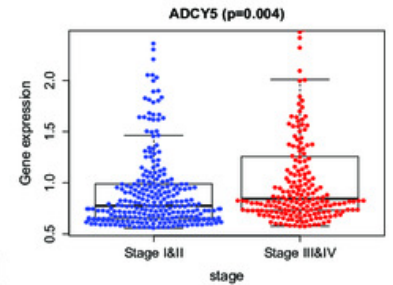

H

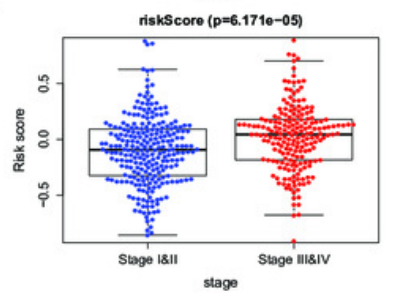

E

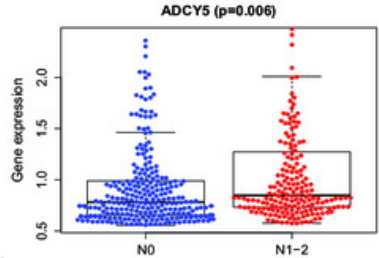

I

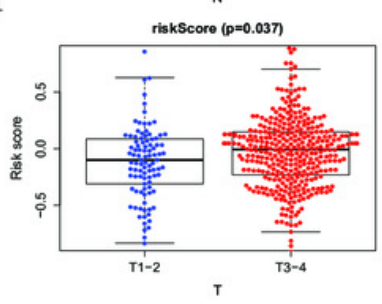

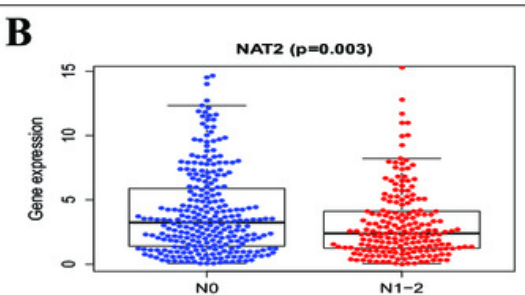

F

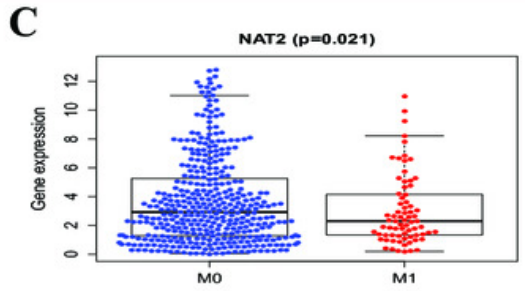

G

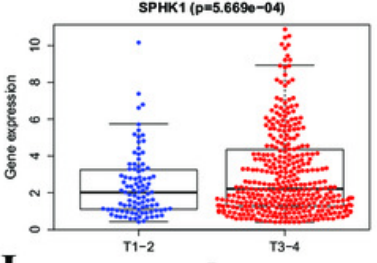

J

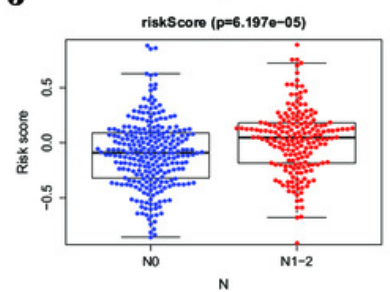

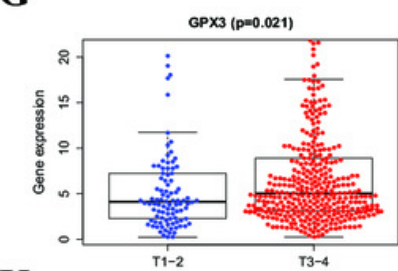

K

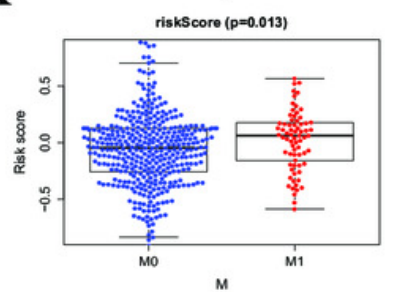

GEO

L

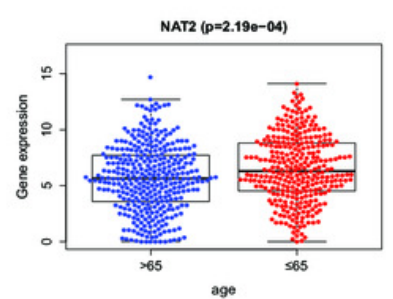

P

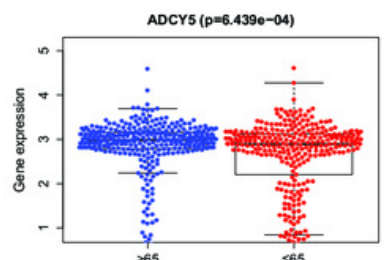

T

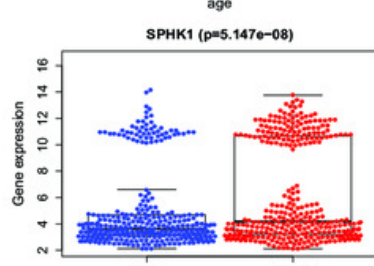

X

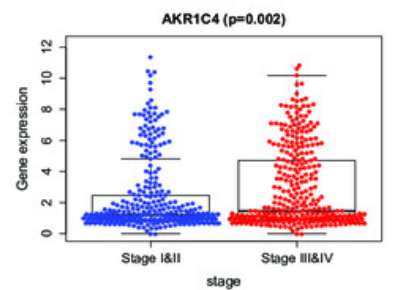

M

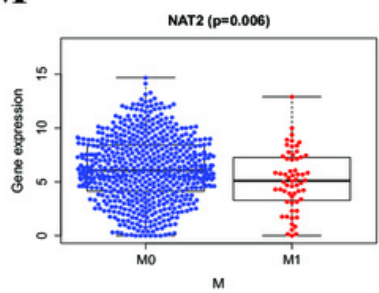

Q

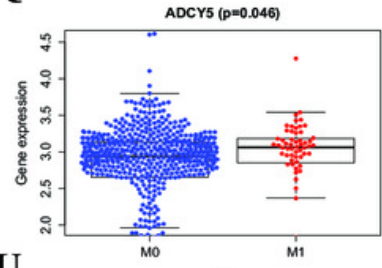

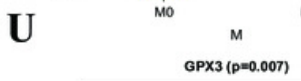

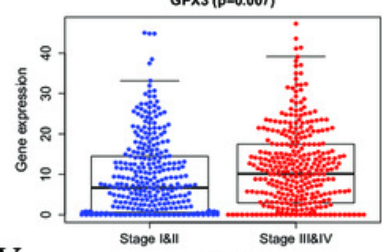

$\mathbf{Y}$

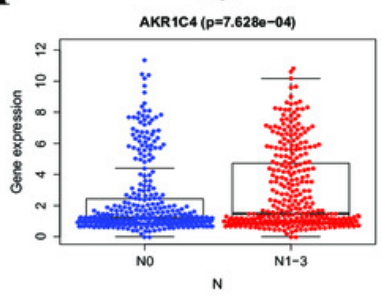

N

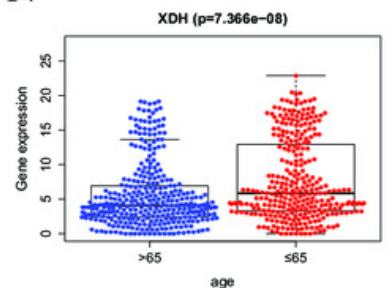

$\mathbf{R}$

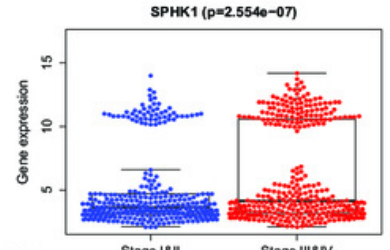

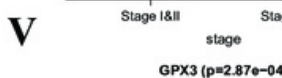

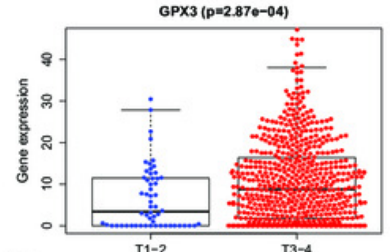

$\mathbf{Z}$

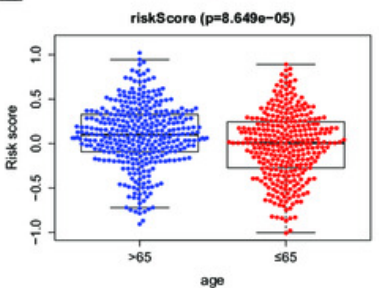

O

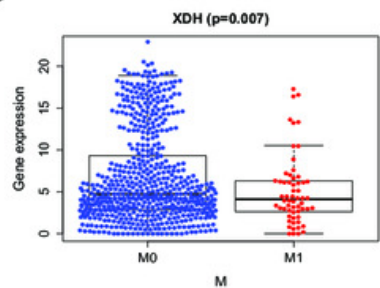

S

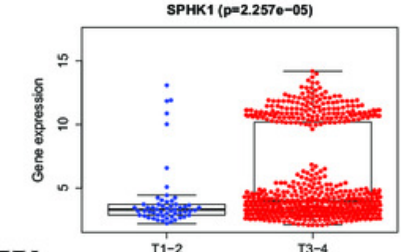

W

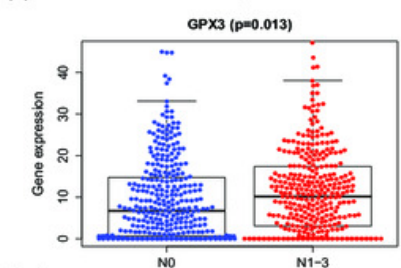

AA

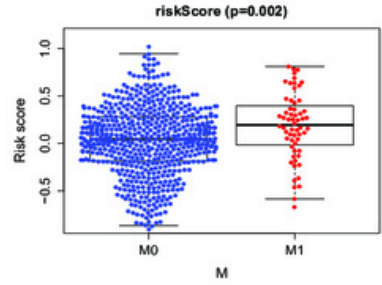




\section{Figure 6}

Figure 6. GO, KEGG, and GSEA analysis.

(A) GO analysis of six PRMGs, on the left of circle chart is the gene, up-regulation was red, and down-regulation was blue, on the right of circle chart is different GO terms, and the genes linked via ribbons to their assigned terms. $(p<0.05)$ (B) KEGG pathway of PRMGs. (C) GSEA analysis of the differentially expressed genes between high and low-risk groups. Green line chart representation enrichment profile, horizontal axis is each gene under the KEGG pathway, and the vertical axis is the corresponding accumulated enrichment score. The peak in the line graph is the enrichment score of this pathway, and the gene before the peak is the core gene of the pathway; Hits representation mark the genes of the pathway with black lines; Ranking metric scores indicates the distribution of rank values of all genes in the pathway. (D) Multiple GSEA analyses of the differentially expressed genes between high and low-risk groups. GO, gene ontology, KEGG, kyoto encyclopedia of genes and genomes, GSEA, gene set enrichment analysis. PRMGs, prognosis-related metabolic genes. 
A

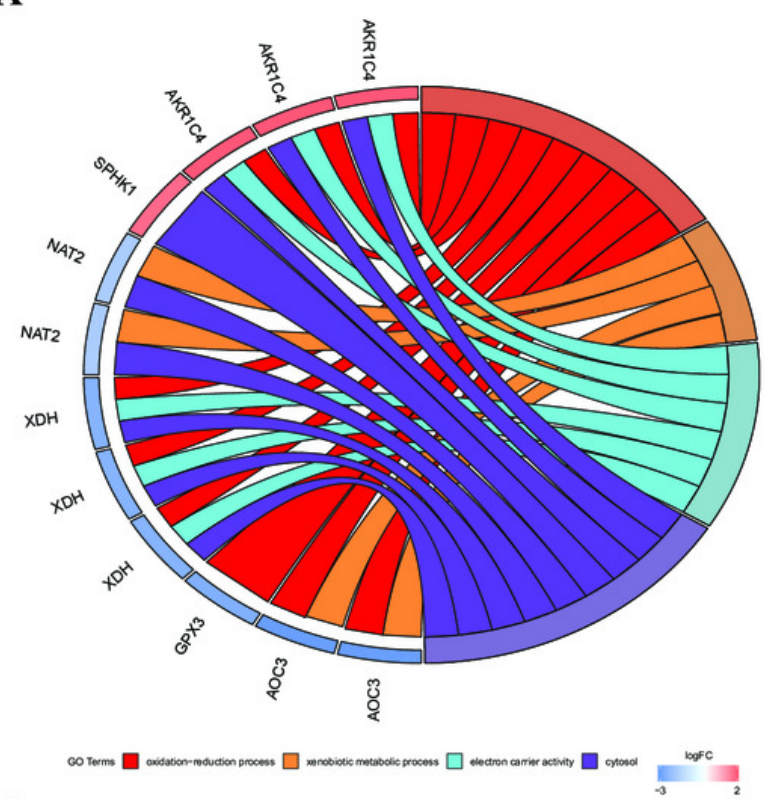

B

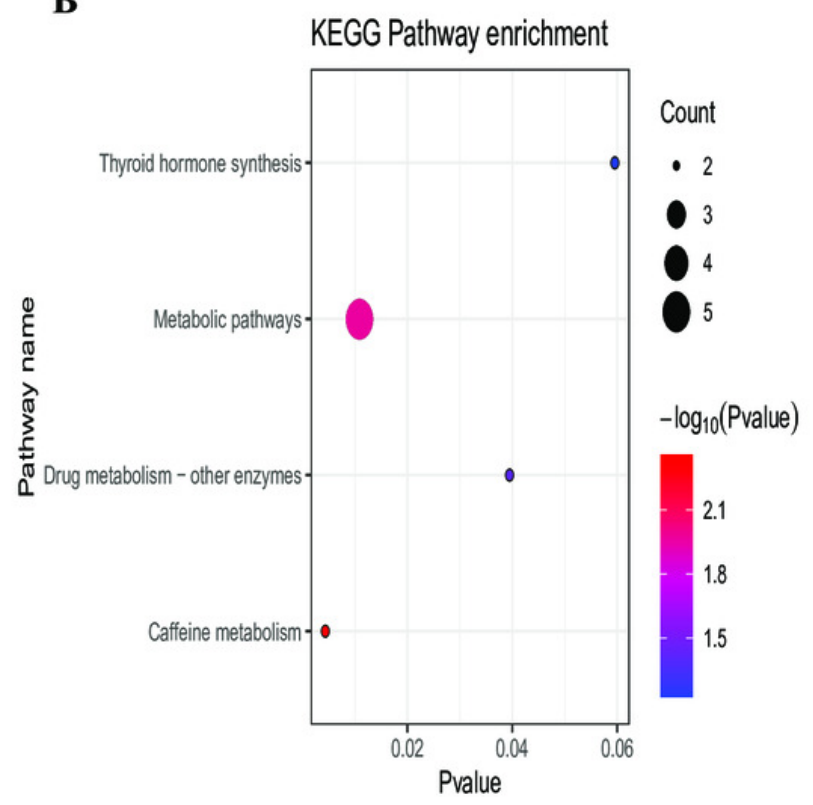

C

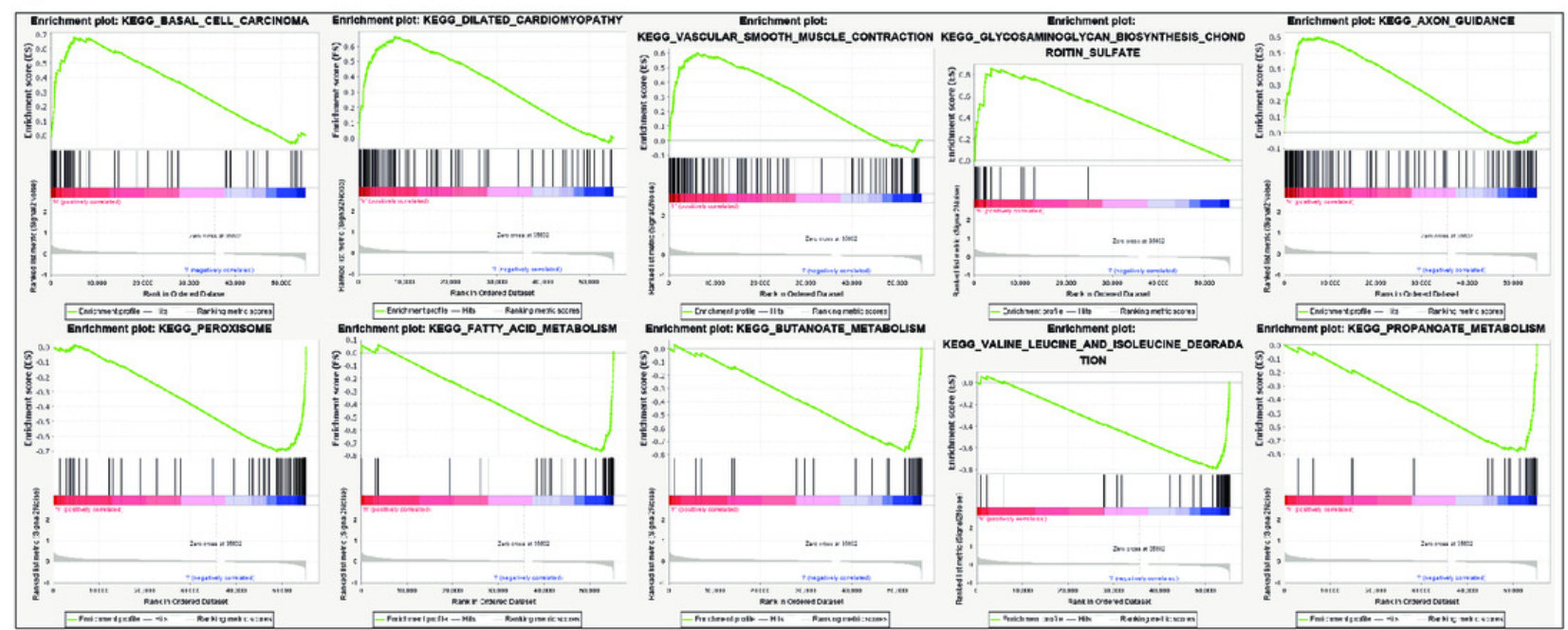

D

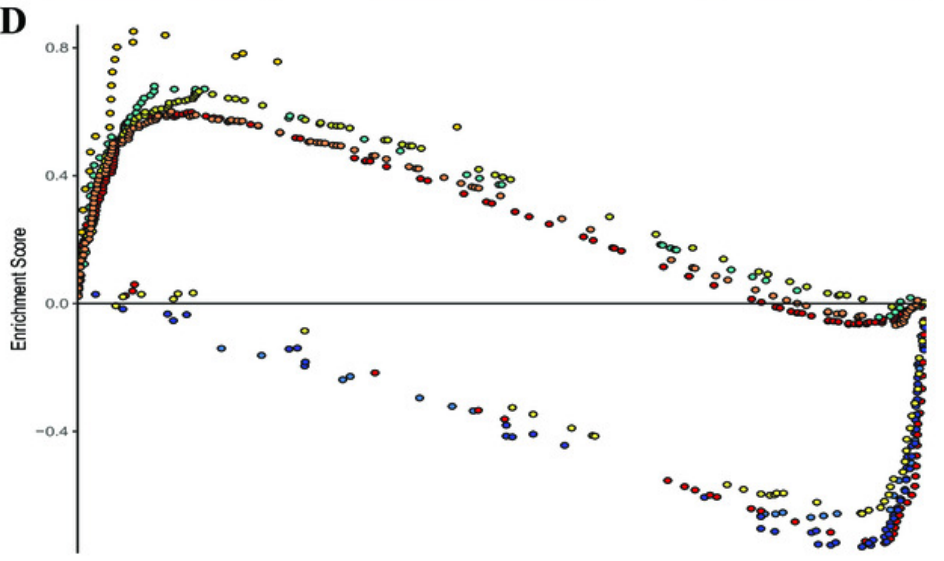

- KEgG_Ascorbate_AND_AldDARATE_METABOlism

- KEgG_aXon_guidANCE

- KEgG_BASAL_CELL_CARCINOMA

- kegg_butanoate_metabolism

- KEgG_dilated cardiomyopathy

- KEgG_FatTY_ACID_METABOLISM

- KEGG_GLYCOSAMINOGLYCAN_BIOSYNTHESIS_CHONDROITIN_SULFATE

- KEgG_PROPANOATE_METABOLISM

- KEgg_VASCULAR_SMOOTH_MUSCLE_CONTRACTION

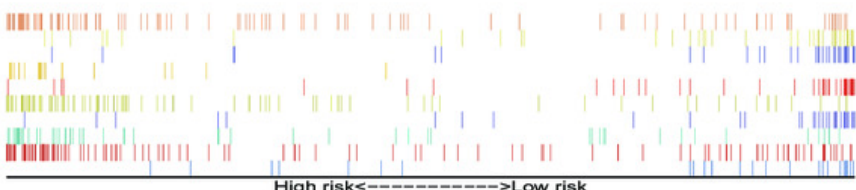




\section{Figure 7}

Figure 7. The nomogram to anticipate prognostic probabilities in CRC.

(A) The nomogram for predicting 1-, 3- and 5-year OS of CRC by clinical-pathological features and Risk score. The 1-, 3- and 5- year calibration curves of TCGA-CRC (B, D, F) and GEO-CRC (C, E, G). $X$ axis represents predicted survival time and $Y$ axis indicates actual survival time. TCGA, the cancer genome atlas database; GEO, gene expression omnibus; CRC, colorectal cancer. OS, overall survival. 
$\mathbf{A}$
Points
ago
gender
stago
$\mathrm{T}$
N
M
riskScoro
Total Points
1-yoar survival
3-year survival
5-year survival

B

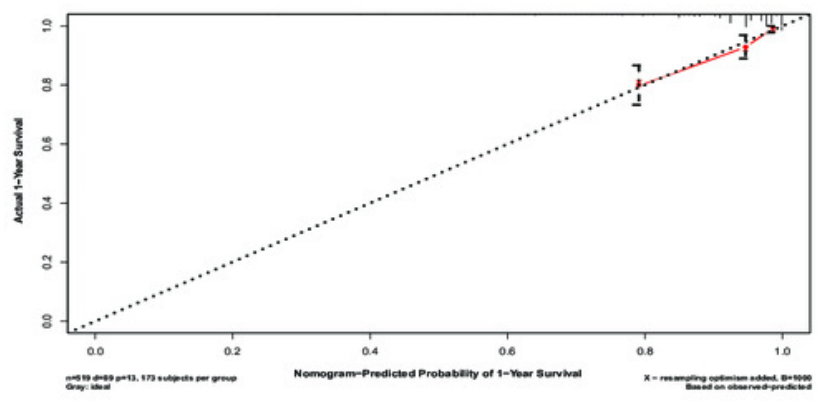

D

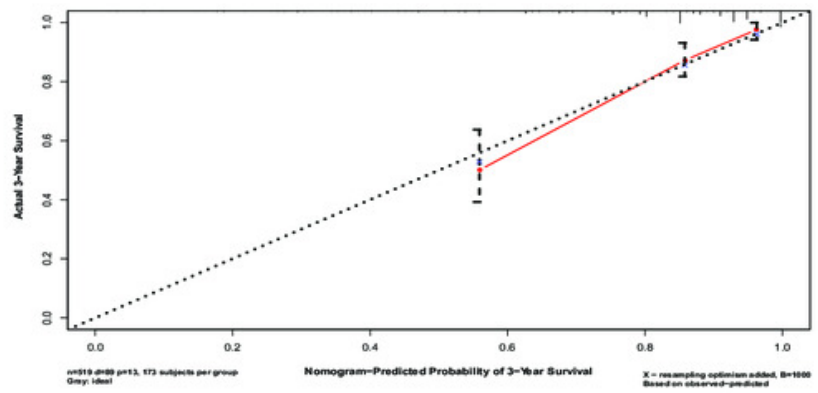

$\mathbf{F}$

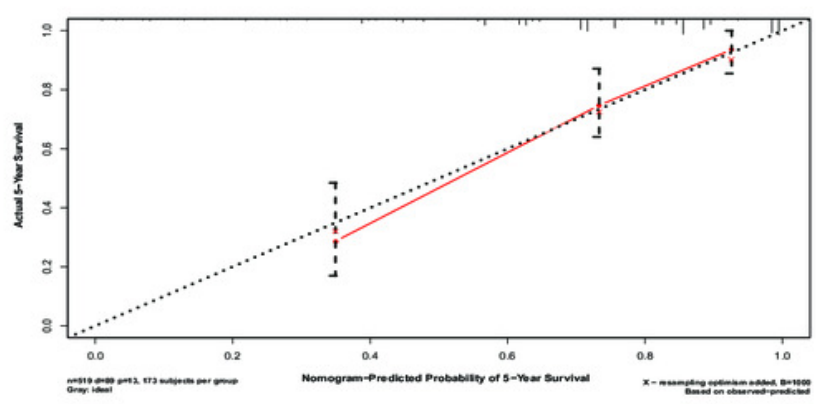

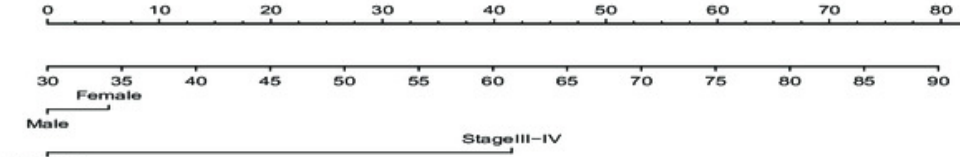
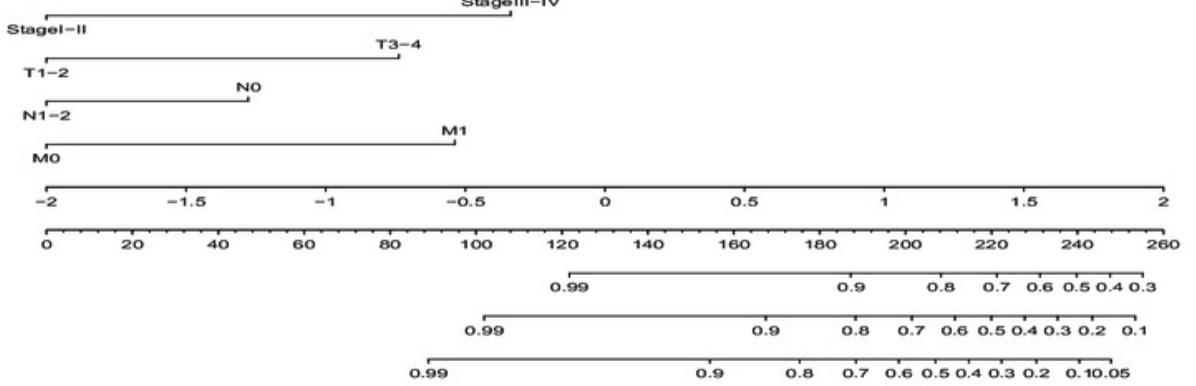

C

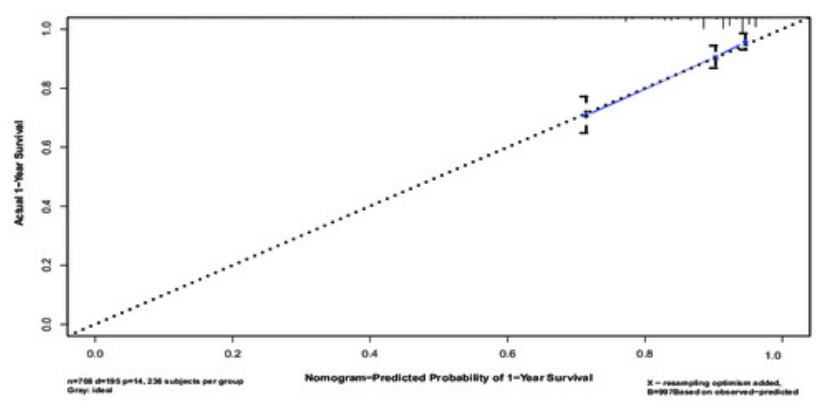

$\mathbf{E}$

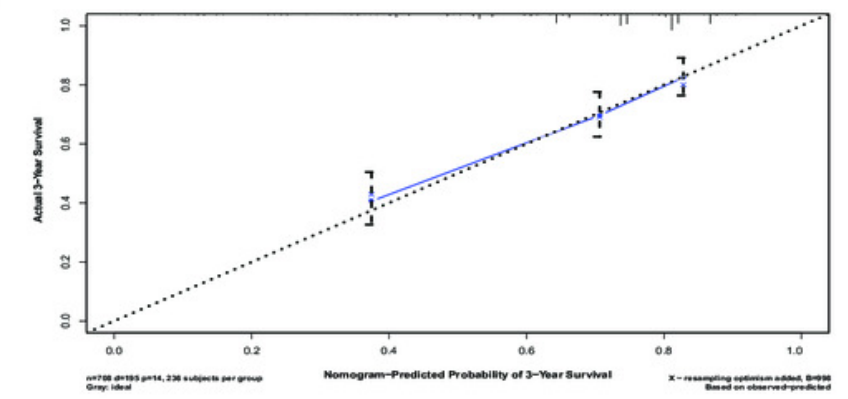

G

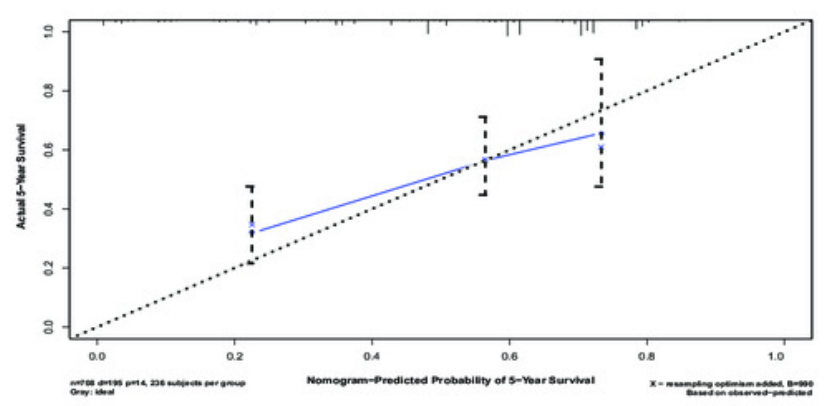

